\title{
Oversampled A/D Conversion and Error-Rate Dependence of Nonbandlimited Signals With Finite Rate of Innovation
}

\author{
Ivana Jovanović, Student Member, IEEE, and Baltasar Beferull-Lozano, Member, IEEE
}

\begin{abstract}
We study the problem of $\mathrm{A} / \mathrm{D}$ conversion and error-rate dependence of a class of nonbandlimited signals with finite rate of innovation. In particular, we analyze a continuous periodic stream of Diracs, characterized by a finite set of time positions and weights. Previous research has only considered sampling of this type of signals, ignoring the presence of quantization, necessary for any practical implementation. To this end, we first define the concept of consistent reconstruction and introduce corresponding oversampling in both time and frequency. High accuracy in a consistent reconstruction is achieved by enforcing the reconstructed signal to satisfy three sets of constraints, related to low-pass filtering, quantization and the space of continuous periodic streams of Diracs. We provide two schemes to reconstruct the signal. For the first one, we prove that the estimation mean squared error of the time positions is $O\left(1 / R_{t}^{2} R_{f}^{3}\right)$, where $R_{t}$ and $R_{f}$ are the oversampling ratios in time and frequency, respectively. For the second scheme, it is experimentally observed that, at the cost of higher complexity, the estimation accuracy lowers to $O\left(1 / R_{t}^{2} R_{f}^{5}\right)$. Our experimental results show a clear advantage of consistent over nonconsistent reconstruction. Regarding the rate, we consider a threshold crossing based scheme where, as opposed to previous research, both oversampling in time and in frequency influence the coding rate. We compare the error-rate behavior resulting, on the one hand, from increasing the oversampling in time and/or frequency, and, on the other hand, from decreasing the quantization stepsize.
\end{abstract}

Index Terms-Consistency, convexity, finite rate of innovation, oversampling, projection, quantization, threshold crossing encoding.

\section{INTRODUCTION}

$\mathbf{R}$ ECENT results in sampling theory [1] have shown that it is possible to develop exact sampling schemes for a certain set of nonbandlimited signals, characterized by having a fi-

Manuscript received August 11, 2004; revised June 8, 2005. This work was supported partially by the National Competence Center in Research on Mobile Information and Communications Systems (NCCR-MICS), a center supported by the Swiss National Science Foundation by Grant 5005-67322. The material in this paper was presented in part at the IEEE International Conference on Acoustics, Speech and Signal Processing, 2004, Montreal, Canada, and the IEEE International Symposium on Information Theory, 2004, Chicago, IL. The associate editor coordinating the review of this manuscript and approving it for publication was Dr. Yuan-Pei Lin.

I. Jovanović is with the Audiovisual Communications Laboratory (LCAV), Ecole Polytechnique Fédérale de Lausanne (EPFL), Lausanne, Switzerland (e-mail: ivana.jovanovic@epfl.ch).

B. Beferull-Lozano was with the Swiss Federal Institute of Technology-EPFL, Lausanne, Switzerland. He is now with the Group of Information and Communication Systems, Instituto de Robotica, Escuela Politecnica Superior de Ingenieria (ETSI), Universidad de Valencia (UV), Valencia, Spain (e-mail: baltasar.beferull@uv.es).

Digital Object Identifier 10.1109/TSP.2006.874363 nite number of degrees of freedom per unit time, which is called finite rate of innovation. Taking a finite number of uniform samples, obtained from an appropriate sampling kernel, we are able to achieve perfect reconstruction. Some of these signals with finite rate of innovation, such as streams of Diracs, have found several applications in CDMA [2], UWB [3] and sensor field sampling [4]. For example, results in [1] can be applied to the problem of multipath delay estimation in wideband channels. On the other hand, in the context of sensor networks measuring physical phenomena, such as temperature, local heat sources can be well modeled by Diracs and the sampling kernel in this case is given by the Green's function of the heat diffusion equation [5]. In [1]-[3], it was assumed that we have no quantization of the acquired samples. However, in any practical application quantization is required. An irreversible loss of information, introduced by quantization makes perfect reconstruction no longer possible. Motivated by the need of quantization, we investigate analog-to-digital (A/D) conversion and the error-rate dependence of nonbandlimited signals with finite rate of innovation, which has not been considered in previous research.

In this paper, we focus on the A/D conversion of a particular class of signals with finite rate of innovation, namely, continuous periodic stream of $K$ Diracs, characterized by a set of time positions $\left\{t_{k}\right\}_{k=0}^{K-1}$ and weights $\left\{c_{k}\right\}_{k=0}^{K-1}$. We study the reconstruction quality of time positions under the presence of quantization. There are two reasons for this: 1) it can be shown that the error in weights depends on the error in time positions, and 2) in many applications, such as UWB and sensor field sampling, the most important information is contained in the positions of pulses.

High reconstruction accuracy in time positions can be achieved by introducing two types of oversampling: 1) oversampling in frequency, determined by the bandwidth extension of the low-pass sampling kernel, and 2) oversampling in time, determined by the number of samples taken from the acquired filtered signal. Introducing the oversampling is equivalent to introducing a redundancy in the system, which usually reduces the sensitivity to degradations. Although this idea is very intuitive, the question of fully exploiting that redundancy is not always simple. This can be already observed in the case of A/D conversion of bandlimited signals, where the simple linear reconstruction is not optimal. For example, in this case, the quantized samples of the original and the reconstructed signal are not necessarily the same, implying a larger reconstruction error on average. The key idea to achieve high accuracy is to have a reconstruction that is consistent with all available knowl- 
edge about the signal and the acquisition process. Thus, in our paper, we explore the concept of consistency by enforcing the reconstructed signal to satisfy three sets of constraints which are related to: low-pass filtering, quantization, and the space of continuous periodic streams of Diracs. A signal reconstruction satisfying the three sets is said to provide Strong consistency while if it satisfies only the first two sets is said to provide Weak consistency.

The concept of consistent reconstruction and the corresponding reconstruction accuracy for the case of bandlimited signals has been considered in [6]-[8]. However, there are three essential differences with our paper: 1) we consider the reconstruction accuracy related to the nonbandlimited signal; 2 ) we exploit the knowledge about the structure of the nonbandlimited signal; 3) we introduce oversampling in frequency in addition to oversampling in time.

In this paper, reconstruction algorithms for both Weak and Strong consistency are proposed. As a quantitative characterization of the reconstruction quality, we consider the mean squared error (MSE) of the time positions and its dependence on the oversampling in time and in frequency. We focus on the MSE related to time positions because, as we show in this paper, the MSE related to the weights of the Diracs depends fundamentally on the MSE of time positions. For the first algorithm, we show both theoretically and experimentally that the MSE performance for the time positions decreases as $O\left(1 / R_{t}^{2} R_{f}^{3}\right)$, where $R_{t}$ and $R_{f}$ are the oversampling ratios in time and frequency, respectively. For the second algorithm, which achieves Strong consistency but at the cost of higher complexity, we obtain experimentally an MSE performance of the order of $O\left(1 / R_{t}^{2} R_{f}^{5}\right)$ [9]. Both results show a clear outperformance of consistent reconstructions over nonconsistent reconstructions.

Regarding the rate, we consider two encoding schemes: a threshold crossing (TC) based scheme, similar to the one proposed in [10] and a PCM encoding scheme. We compare the error-rate dependence that is obtained, on the one hand, from both increasing the oversamplings in time and in frequency, and on the other hand, from decreasing the quantization stepsize. The main novelty of the TC encoding analysis, introduced in our paper, is the additional dependence of the maximal number of threshold crossings on the oversampling in frequency. This comes as a consequence of considering nonbandlimited signals with finite rate of innovation. Our results show that, using the TC encoding, we can achieve the same error-rate dependence, by a) increasing the oversampling in time and b) decreasing the quantization stepsize [11]. This is very important from a practical point of view because the cost of halving the quantization stepsize is much higher than that of doubling any of the oversampling ratios (complex and expensive analog circuitry). Moreover, in order to make the TC encoding scheme work, in our case, we can adjust three parameters (the quantization stepsize and the two oversamplings), as compared to the case of bandlimited signal [10], where only two parameters are adjusted (the quantization stepsize and the oversampling in time).

Although our theoretical analysis is restricted to periodic streams of Diracs, the algorithms proposed in this paper can be also used for reconstructing other signals with finite rate of innovation, such as finite streams of Diracs and nonuniform splines.
This paper is organized as follows. Section II introduces the class of signals given by continuous-time periodic streams of Diracs. Section III defines the oversampling in time and in frequency. Section IV introduces the concept of Weak consistency and Strong consistency and proposes the corresponding reconstruction algorithms. In Section V, we prove an upper bound for the MSE performance achieved by Weak consistency and in Section VI, we present the experimental results on the reconstruction quality for both Weak and Strong consistency. In Section VII and Section VIII, we describe and analyze the threshold crossing based encoding and address the rate and error-rate dependence. We compare the error-rate dependence as a function of the both oversamplings and the quantization stepsize. Finally, in Section IX, we conclude with a brief summary of our paper and directions for future work.

\section{Signals With Finite Rate of InNOVATION}

New results on sampling theory show that certain classes of nonbandlimited signals, such as periodic and finite length streams of Diracs, nonuniform splines and piecewise polynomials, can be perfectly reconstructed from a finite number of uniform samples, using sinc and Gaussian sampling kernels. Intuitively, these classes of signals are characterized by having a finite number of degrees of freedom per unit of time, namely, having a finite rate of innovation.

In this paper, we consider a periodic stream of $K$ Diracs, that is,

$$
x(t)=\sum_{k \in \mathbb{Z}} c_{k} \delta\left(t-t_{k}\right)
$$

with period $\tau$, where $t_{k+K}=t_{k}+\tau, c_{k+K}=c_{k}, \forall k \in \mathbb{Z}$, and $\delta(t)$ denotes a Dirac delta function. This signal has $2 K / \tau$ degrees of freedom per unit of time, since the only knowledge that is required to determine the signal uniquely is given by the $K$ time positions $\left\{t_{k}\right\}_{k=0}^{K-1}$ and the $K$ weights $\left\{c_{k}\right\}_{k=0}^{K-1}$. A periodic stream of $K$ Diracs $x(t)$ can be represented through its Fourier series, as follows:

$$
x(t)=\sum_{m \in \mathbb{Z}} X[m] e^{j \frac{2 \pi m t}{\tau}}, \text { where } X[m]=\frac{1}{\tau} \sum_{k=0}^{K-1} c_{k} e^{-j \frac{2 \pi m t_{k}}{\tau}} .
$$

After sampling the signal with the sinc sampling kernel, $h_{B}(t)=B \operatorname{sinc}(B t), B \tau=2 M+1 \geq 2 K+1$, thus obtaining $y(t)-x(t) * h_{B}(t)$, the uniform samples of $y(t)$ are given by

$$
y_{n}=\sum_{m=-M}^{M} X[m] e^{j \frac{2 \pi m n}{N}} \text { where } \quad N \geq 2 M+1 .
$$

Taking at least $2 K+1$ samples $\left\{y_{n}\right\}_{n=1}^{2 K+1}$, we can directly compute the $2 K+1$ Fourier coefficients $X[m]$, using (2). From $2 K+1$ Fourier coefficients $X[m]$, we can reconstruct first time positions and then weights. Thus, it is clear that low-pass version of the original signal $x(t)$, that we call $y(t)$, is sufficient for the signal reconstruction. Also, we notice that Fourier coefficients $X[m]$ of the signal $x(t)$ coincides with discrete-time Fourier series (DTFS) of $y_{n}$, i.e., $X[m]=Y[m]$, and for this 


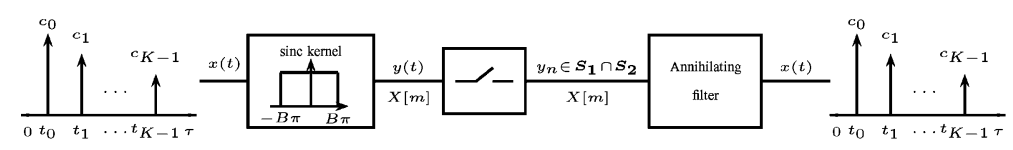

(a)

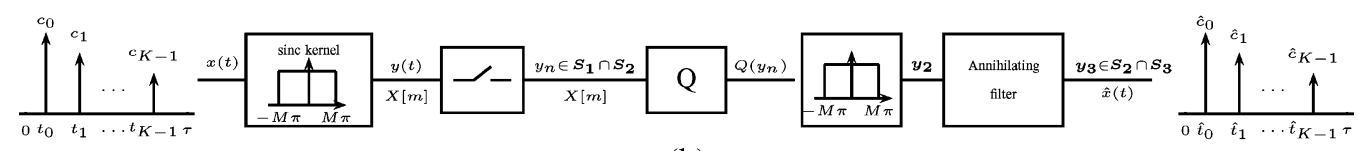

(b)

Fig. 1. Reconstruction algorithms for a periodic stream of Diracs. (a) Without introducing quantization. (b) Introducing quantization of the samples $y_{n}$. The annihilating filter in (a) corresponds to (3) and the one in (b) corresponds to (4).

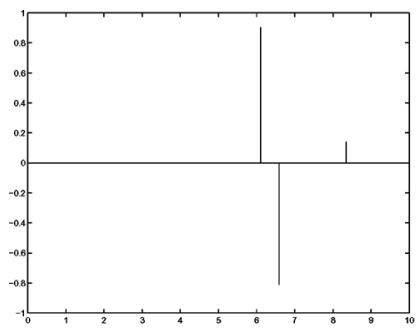

(a)

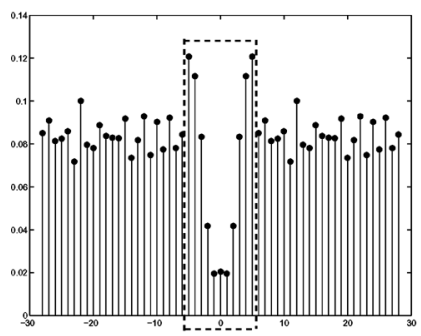

(e)

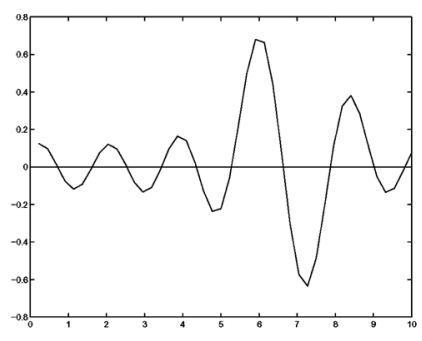

(b)

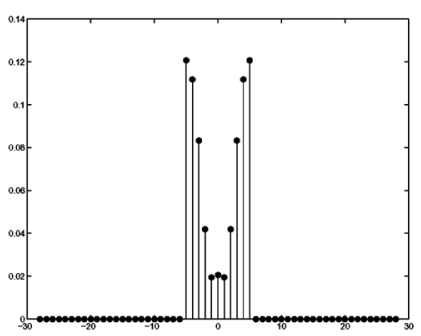

(f)

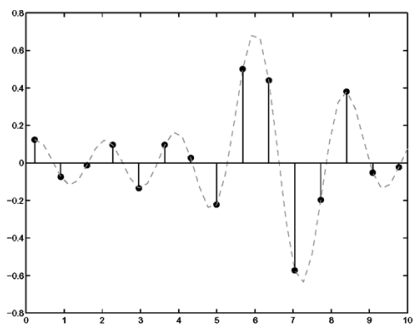

(c)

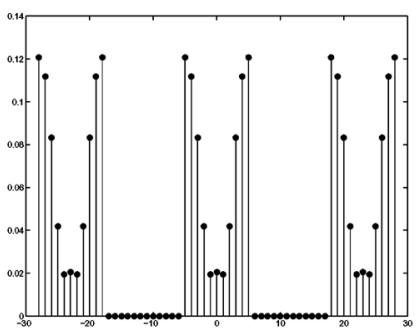

(g)

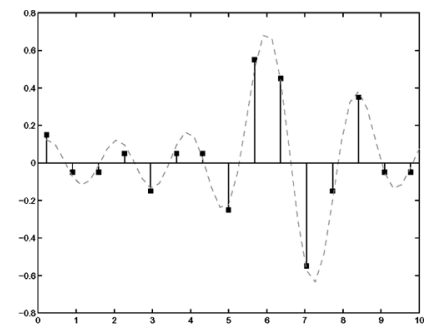

(d)

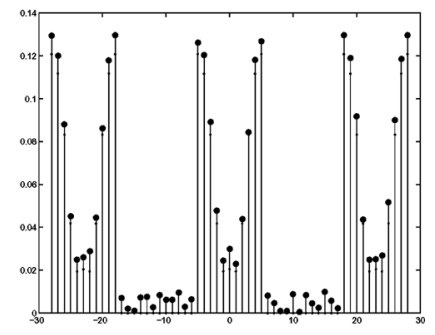

(h)

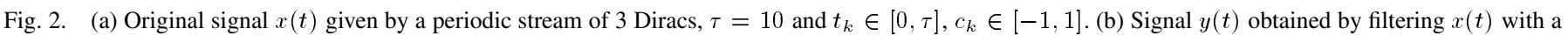

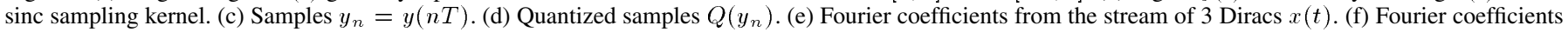

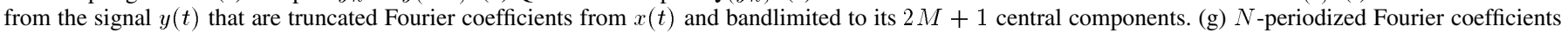

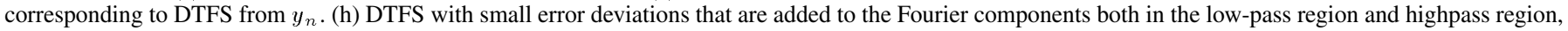
thus making the perfect reconstruction no longer possible.

reason, later, when talking about the signal $y(t)$ we will usually refer to $Y[m]$. Analyzing the Fourier components in (1), it can be seen that each exponential term $u_{k}=e^{-j\left(2 \pi t_{k} / \tau\right)}$ can be annihilated by a first order FIR annihilating filter $A_{k}(z)=$ $\left(1-e^{-j\left(2 \pi t_{k} / \tau\right)} z^{-1}\right)$. Extension of the filter order to $K$ results in a filter $A(z)=\prod_{k=1}^{K}\left(1-e^{-j\left(2 \pi t_{k} / \tau\right)} z^{-1}\right)$ that annihilates all Fourier coefficients. In matrix notation, this can be represented as

$$
\left(\begin{array}{cccc}
X[0] & X[-1] & \ldots & X[-K] \\
X[1] & X[0] & \ldots & X[-(K-1)] \\
\vdots & \vdots & \ddots & \vdots \\
X[K] & X[K-1] & \ldots & X[0]
\end{array}\right)\left(\begin{array}{c}
a_{0} \\
a_{1} \\
\vdots \\
a_{K}
\end{array}\right)=\left(\begin{array}{c}
0 \\
0 \\
\vdots \\
0
\end{array}\right)
$$

where $a_{i}$ is the $i$ th coefficient of the annihilating polynomial. Thus, if we are given the $2 K+1$ exact Fourier coefficients, by setting $a_{0}=1$, we can find the unique solution of (3). The roots of the annihilating filter $A(z),\left\{u_{k}=e^{-j\left(2 \pi t_{k} / \tau\right)}\right\}_{k=0}^{K-1}$, reveal the $K$ time positions $\left\{t_{k}\right\}_{k=0}^{K-1}$, while the corresponding weights $\left\{c_{k}\right\}_{k=0}^{K-1}$ can be then directly computed from (1) [see Fig. 1(a)].

Notice that all previous steps assume no quantization in amplitude. Hence, no error in $\boldsymbol{y}=\left[y_{0}, \ldots, y_{N-1}\right]^{T}$ ensures the existence of the previous exact solution. In our work we study A/D conversion and thus we consider the operation of quantization performed on $\boldsymbol{y}$ [see Fig. 1(b)]. The quantization error causes an irreversible loss of information and makes the exact recovery of $x(t)$ no longer possible (see Fig. 2). In order to overcome this problem, as the first step, we are going to introduce two types of oversampling.

\section{OVERSAMPLING IN TIME AND FREQUENCY}

We consider two types of oversampling to compensate the error introduced by quantization. The first one consists of taking more samples $\boldsymbol{y}$ than we need, or equivalently, taking samples of $y(t)$ above the Nyquist rate. In that case, we have $N>2 M+1$. This introduces an oversampling in time, which is characterized by oversampling ratio $R_{t}=N /(2 M+1)$.

Notice that we can also perform an additional type of oversampling by extending the bandwidth of the sampling kernel to be greater than the rate of innovation, or equivalently, making $2 M+1>2 K+1$. We denote this type of oversampling as an oversampling in frequency with the oversampling ratio $R_{f}=(2 M+1) /(2 K+1)$. As explained in Section IV, the oversampling in frequency will modify the annihilating filter 
method illustrated in (3), and the corresponding matrix has to be augmented because we use more Fourier coefficients.

We remark also that the number of samples is always proportional to $R_{t}$ and $R_{f}$ since $N=(2 M+1) R_{t}=(2 K+1) R_{f} R_{t}$. This means that $N$ increases linearly with both types of oversampling. As shown in Sections V and VI, by increasing these two oversamplings, and using proper reconstruction schemes, we can substantially increase the reconstruction accuracy.

\section{CONSISTEnt ReCOnStRUCTION}

In the reconstruction process, we enforce the concept of consistent reconstruction, previously introduced in [6] for the case of bandlimited signals. The idea of consistent reconstruction is to exploit all the knowledge from both the a priori properties of the original signal and the information provided by the quantization process. Thus, the key is to find a reconstruction which is consistent with all the available knowledge. Intuitively, a consistent reconstruction will provide, on average, a better reconstruction accuracy than a nonconsistent reconstruction.

We first define all the properties that a reconstruction should satisfy in order to be consistent. Each property defines a set of signals, thus, requiring the satisfaction of a certain property is equivalent to requiring the membership in a certain set. The fact that all properties are satisfied by the original signal ensures that the corresponding sets have a nonempty intersection. All the constraints, or equivalently, the sets, are going to be defined as a subsets of the space of $N$-periodic discrete-time signals, that we call $\mathcal{H}$.

The first set of constraints $\boldsymbol{S}_{\mathbf{1}}$ is related to the quantization operation. The samples $\boldsymbol{y}$ are uniformly quantized, as

$$
y_{n}^{q}=Q\left(y_{n}\right)=\Delta\left(\left\lfloor y_{n} / \Delta\right\rfloor+1 / 2\right)
$$

where $\Delta$ is the quantization stepsize. ${ }^{1}$ Let $l_{n}=$ $\left[\Delta\left\lfloor y_{n} / \Delta\right\rfloor, \Delta\left\lfloor y_{n} / \Delta\right\rfloor+\Delta\right]$ be the quantization interval to which the sample $y_{n}$ belongs. The quantized samples $y_{n}^{q}$ give the information about the intervals in which all the samples $y_{n}$ lie. The set of these intervals is an $N$-dimensional cube. More precisely:

Set $\boldsymbol{S}_{\mathbf{1}}$ : Given $\boldsymbol{y}$ and $\boldsymbol{y}^{\boldsymbol{q}}=\mathbb{Q}(\boldsymbol{y})=\left[y_{0}^{q}, \ldots, y_{N-1}^{q}\right]^{T}$, the set $S_{1}=\mathbb{Q}^{-1}\left(\boldsymbol{y}^{q}\right)$ defines a convex set of sampled signals such that all of them are quantized to the same quantization bins [see Fig. 3(a)].

The second set of constraints $\boldsymbol{S}_{\mathbf{2}}$ comes from the fact that the signal $y(t)$, obtained after filtering $x(t)$, is periodic and bandlimited.

Set $\boldsymbol{S}_{2}$ : The set $\boldsymbol{S}_{\mathbf{2}}$ is a set of $N$-periodic discrete-time signals bandlimited to $2 M+1$ nonzero DTFS components.

In addition to the fact that the $N$-periodic discrete-time signals should have $2 M+1$ nonzero DTFS components we also want to use the knowledge that $x(t)$ is a periodic stream of Diracs. Therefore, we define another set of constraints, as follows.

Set $\boldsymbol{S}_{3}$ : The set $\boldsymbol{S}_{3}$ is a set of $N$-periodic discrete-time signals, such that the $\{Y[m]\}_{m=-M}^{M}$ DTFS components originate from a periodic stream of Diracs. This amounts

\footnotetext{
${ }^{1}$ We could equivalently define another type of quantizer, as $y_{n}^{q}=Q\left(y_{n}\right)=$ $\Delta\left\lfloor y_{n} / \Delta\right\rfloor$, since the choice of the quantizer is not going to have influence on our results.
}

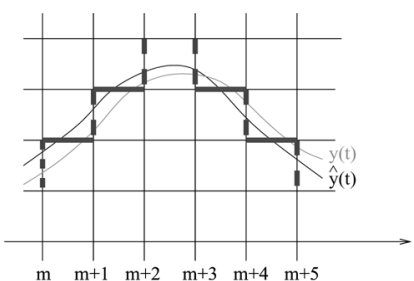

(a)

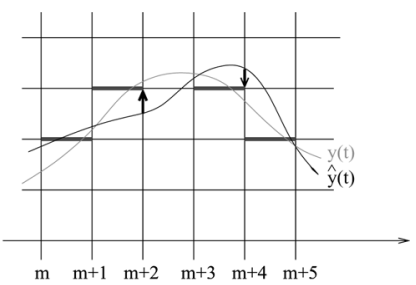

(b)
Fig. 3. (a) The estimated function $\hat{y}(t)$ is consistent with the original signal $y(t)$ with respect to the quantization bins. (b) If the estimated function $\hat{y}(t)$ is not consistent with $y(t)$, then we project to the border of the corresponding quantization bin.

to having $Y[m]=X[m]=(1 / \tau) \sum_{k=0}^{K-1} c_{k} e^{-j 2 \pi m t_{k} / \tau}$, $m=-M, \ldots, M$, with $c_{k} \in \mathbb{R}, 0<t_{k} \leq \tau$, while there are no constraints on $Y[m]$ for $|m|>M$.

We can get more insight into the structure of the sets $\boldsymbol{S}_{1}, \boldsymbol{S}_{2}$ and $\boldsymbol{S}_{\mathbf{3}}$ if we observe that $\boldsymbol{S}_{\mathbf{1}}$ is an $N$-dimensional hypercube in the $N$-dimensional space $\mathcal{H}, \boldsymbol{S}_{\mathbf{2}}$ is an $(2 M+1)$-dimensional subspace of $\mathcal{H}$, and that $\boldsymbol{S}_{\boldsymbol{2}} \cap \boldsymbol{S}_{\boldsymbol{3}}$ is a $(2 K)$-dimensional (nonlinear) surface inside $\boldsymbol{S}_{2}$. This dimensional argument naturally brings the notation of oversampling by space dimension ratios: $N /(2 M+1)$ and $(2 M+1) /(2 K)$.

Now, we are going to define projections on the corresponding sets.

Projection $\boldsymbol{P}_{\mathbf{1}}$ : Given a set of samples $\boldsymbol{y}, \boldsymbol{y}_{\mathbf{1}}=\boldsymbol{P}_{\mathbf{1}}(\boldsymbol{y})$ is obtained as

1) if $y_{n} \in \boldsymbol{S}_{\mathbf{1}}$, then $y_{1, n}=y_{n}$.

2) else, $y_{1, n}$ is taken to be equal to the closest border of the quantization interval $Q^{-1}\left(y_{n}^{q}\right)$, that is

$$
\boldsymbol{P}_{\mathbf{1}}\left(y_{n}\right)=y_{n}^{q}+\operatorname{sign}\left(y_{n}-y_{n}^{q}\right)(\Delta / 2) .
$$

Projection $\boldsymbol{P}_{\mathbf{2}}$ : Given an $N$-periodic discrete time signal $\boldsymbol{y}, \boldsymbol{y}_{2}=\boldsymbol{P}_{2}(\boldsymbol{y})$ is obtained by low-pass filtering, such that the nonzero DTFS components are $Y_{2}[m]=Y[m]$ for $m=-M, \ldots, M$.

Projection $\boldsymbol{P}_{3}$ : Given an $N$-periodic discrete time signal $\boldsymbol{y}$, the projection $\boldsymbol{P}_{\mathbf{3}}$ provides a new signal $\boldsymbol{y}_{\mathbf{3}}=\boldsymbol{P}_{\mathbf{3}}(\boldsymbol{y})$, with the set of in-band DTFS $\left\{Y_{3}[m]\right\}_{m=-M}^{M}$ that are $Y_{3}[m]=(1 / \tau) \sum_{k=0}^{K-1} c_{k} e^{-j\left(2 \pi m t_{k} / \tau\right)}$, with $c_{k} \in \mathbb{R}$ $0<t_{k} \leq \tau$ while the out-band DTFS remain the same, i.e., $Y_{3}[m]=Y[m]$ for $|m|>M$.

Projection $P_{3}$ involves augmenting the matrix in (3) using $2 M+1$ Fourier components. Notice that, since the quantization taking place, we do not have the exact Fourier coefficients, but only estimates $Y_{2}[m]=\hat{X}[m]$, and therefore (3) does not have an exact solution. In order to get better estimates of the time positions, we use a generalized form of (3) with $2 M+1$ Fourier component and increased order of the annihilating filter, as follows:

$$
\left(\begin{array}{cccc}
Y_{2}[0] & Y_{2}[-1] & \cdots & Y_{2}[-L] \\
Y_{2}[1] & Y_{2}[0] & \cdots & Y_{2}[-L+1] \\
\vdots & \vdots & \ddots & \vdots \\
Y_{2}[M] & Y_{2}[M-1] & \cdots & Y_{2}[M-L]
\end{array}\right)\left(\begin{array}{c}
a_{0} \\
a_{1} \\
\vdots \\
a_{L}
\end{array}\right) \simeq\left(\begin{array}{c}
0 \\
0 \\
\vdots \\
0
\end{array}\right)
$$

where the left-hand side matrix has a size $(M+1) \times(L+1)$ with $K \leq L \leq M$ and $L$ is the filter order. In (4), we indicate with $\simeq$ that the system of equation is not exactly satisfied. Notice here 
how the oversampling in frequency is introduced by extending the number of rows from $K+1$ to $M+1$ and at the same time, making the order of the filter larger than $K$. By taking $a_{0}=1$, the system (4) becomes equivalent to a high-order Yule-Walker (HOYW) system [12]

$$
\begin{aligned}
& \left(\begin{array}{cccc}
Y_{2}[-1] & Y_{2}[-2] & \cdots & Y_{2}[-L] \\
Y_{2}[0] & Y_{2}[-1] & \cdots & Y_{2}[-(L-1)] \\
\vdots & \vdots & \ddots & \vdots \\
Y_{2}[M-1] & Y_{2}[M-2] & \cdots & Y_{2}[M-L]
\end{array}\right)\left(\begin{array}{c}
a_{1} \\
a_{2} \\
\vdots \\
a_{L}
\end{array}\right) \\
& \simeq-\left(\begin{array}{c}
Y_{2}[0] \\
Y_{2}[1] \\
\vdots \\
Y_{2}[M]
\end{array}\right)
\end{aligned}
$$

or in matrix notation

$$
\hat{\boldsymbol{H}} \boldsymbol{a} \simeq-\hat{\boldsymbol{h}}
$$

where $\hat{h}=\left[Y_{2}[0] \ldots Y_{2}[M]\right]^{T}, \boldsymbol{a}=\left[a_{1} \ldots a_{L}\right]^{T}$, and

$$
\hat{\boldsymbol{H}}=\left(\begin{array}{cccc}
Y_{2}[-1] & Y_{2}[-2] & \cdots & Y_{2}[-L] \\
Y_{2}[0] & Y_{2}[-1] & \cdots & Y_{2}[-(L-1)] \\
\vdots & \vdots & \ddots & \vdots \\
Y_{2}[M-1] & Y_{2}[M-2] & \cdots & Y_{2}[M-L]
\end{array}\right) .
$$

Since both $\hat{\boldsymbol{H}}$ and $\hat{\boldsymbol{h}}$ are distorted from the original values, the use of total least square (TLS) method, which allows for the fact that both $\hat{\boldsymbol{H}}$ and $\hat{\boldsymbol{h}}$ may have some error, instead of least square (LS) method is more appropriate [13]. Simulation results in [14] show that, in general, for solving HOYW equations the TLS method achieves the better accuracy than the LS method. This is particularly clear in cases where the zeros of the annihilating filter approach the unit circle [13]. As pointed out before, the order $L$ of the annihilating filter may lie between $K$ and $M$. So, there will be $K$ "correct" or signal-related roots and $L-K$ extraneous roots, created artificially by the method. There are several ways to decide the positions of the "correct" roots. We propose the following two methods.

1) Choose the $K$ roots that are closest to the unit circle. This is the common solution used for the problem of retrieval of sinusoids in noise [15], which can be seen as a dual problem.

2) Perform two steps.

a) Compute roots without increasing the filter order.

b) Compute roots increasing the filter order and choose the roots that are the closest to the roots in a).

Notice that by increasing $M$ and $L$, extraneous roots can be very close to the unit circle and the first method might fail. Since the second method does not have this problem and we are primarily interested in the reconstruction accuracy for high oversamplings, we use the second method.

If there was no quantization and the estimated $\left\{Y_{2}(m)\right\}_{m=-M}^{M}$ were the exact ones, then the chosen roots would all lie on the unit circle. However, because of the quantization error, an additional step is required after the TLS projection. It consists of projecting the obtained roots to the unit circle, in order to get unit-norm root estimates $\hat{u}_{k}=e^{-j\left(2 \pi \hat{t}_{k} / \tau\right)}$. From $\hat{u}_{k}$, we can directly compute the time positions $\left\{\hat{t}_{k}\right\}_{k=0}^{K}$. Then, using (1), we can estimate the weights $\left\{\hat{c}_{k}\right\}_{k=0}^{K}$. The whole process including the TLS projection, extracting the "correct" roots and computing the time positions and weights, can be seen as the third projection $\boldsymbol{P}_{\mathbf{3}}$.

Notice that although we are primarily interested in the reconstruction $\hat{x}(t)$, we can also consider the reconstruction $\boldsymbol{y}_{\mathbf{3}} \in$ $S_{2} \cap S_{3}$ since there is one-to-one correspondence between the set of all possible inputs $x(t)$ and a subset of $\mathcal{H}$, which is exactly $S_{2} \cap S_{3}$. After defining the sets of constraints and the corresponding projections we are ready to formally define the nonconsistent reconstruction and to introduce the two levels of consistency.

Definition 1: Reconstruction $\boldsymbol{y}_{\mathbf{3}}=\boldsymbol{P}_{\mathbf{3}}\left(\boldsymbol{P}_{\mathbf{2}}\left(\boldsymbol{y}^{\boldsymbol{q}}\right)\right)$ is called a nonconsistent reconstruction.

What makes this reconstruction nonconsistent is the fact that after resampling and requantizing, the signal $\boldsymbol{P}_{\mathbf{2}}\left(\boldsymbol{y}^{\boldsymbol{q}}\right)$ may not always lie in the same quantization bins as the original $\boldsymbol{y}$, or equivalently, it is possible that $\boldsymbol{P}_{\mathbf{2}}\left(\boldsymbol{y}^{\boldsymbol{q}}\right) \notin \boldsymbol{S}_{\mathbf{1}} \cap \boldsymbol{S}_{\mathbf{2}}$. Notice that, quantization applied to a signal that belongs to $S_{1} \cap S_{2}$ makes it leave $\boldsymbol{S}_{1} \cap \boldsymbol{S}_{2}$, although it still remains in the global space $\mathcal{H}$. Certain improvement can be achieved forcing some of the previously defined constraints. Therefore, we define Weak consistent reconstruction, as follows:

Definition 2: Reconstruction $\boldsymbol{y}_{\mathbf{3}} \in \boldsymbol{P}_{\mathbf{3}}\left(\boldsymbol{S}_{\mathbf{1}} \cap \boldsymbol{S}_{\mathbf{2}}\right)$ is called Weak consistent reconstruction.

To impose the Weak consistent reconstruction, notice that sets $\boldsymbol{S}_{1}$ and $\boldsymbol{S}_{2}$ are convex, and $\boldsymbol{P}_{\mathbf{1}}$ and $\boldsymbol{P}_{\mathbf{2}}$ are convex projections. Therefore, starting from the quantized samples $\boldsymbol{y}^{\boldsymbol{q}}$ obtained from the original signal, and iterating only the projections $\boldsymbol{P}_{\mathbf{1}}$ and $\boldsymbol{P}_{2}$, we will converge to $\boldsymbol{y}_{\mathbf{2}} \in \boldsymbol{S}_{1} \cap \boldsymbol{S}_{2}$. The convergence is ensured by the theorem of alternating projections on convex sets (POCS) [15]. In practice, numerically speaking, a Weak consistent reconstruction can be approached within a finite number of iterations. Once we have converged to the reconstruction $\boldsymbol{y}_{\mathbf{2}} \in$ $\boldsymbol{S}_{1} \cap \boldsymbol{S}_{2}$, we apply the additional projection $\boldsymbol{P}_{\mathbf{3}}$, on the set $\boldsymbol{S}_{\mathbf{3}}$ to obtain $\boldsymbol{y}_{\mathbf{3}} \in \boldsymbol{P}_{\mathbf{3}}\left(\boldsymbol{S}_{\mathbf{1}} \cap \boldsymbol{S}_{\mathbf{2}}\right) \subset \boldsymbol{S}_{\mathbf{2}} \cap \boldsymbol{S}_{\mathbf{3}}$. The Weak consistency algorithm is illustrated in Fig. 4(b).

Following the idea of Weak consistent reconstruction we can extend the concept of consistency to not only the two sets $\boldsymbol{S}_{\mathbf{1}}$ and $\boldsymbol{S}_{2}$ but also to $\boldsymbol{S}_{3}$. These three sets are used to enforce a stronger sense of consistency, that is called Strong consistency, and it is defined as follows:

Definition 3: Reconstruction $\boldsymbol{y}_{\mathbf{3}} \in \boldsymbol{S}_{\mathbf{1}} \cap \boldsymbol{S}_{\mathbf{2}} \cap \boldsymbol{S}_{3}$ is called Strong consistent reconstruction.

The concept of the Strong consistency adds a third property in addition to the previous two properties defined by the concept of Weak consistency. Similarly to the Weak consistency algorithm we can define a Strong consistency algorithm, where we generalize the idea of alternating projections to more than two projections. We form a composite projection by the sequential application of $\boldsymbol{P}_{\mathbf{1}}, \boldsymbol{P}_{\mathbf{2}}$ and $\boldsymbol{P}_{\mathbf{3}}$ and the goal is to converge to a point in the intersection set $\boldsymbol{S}_{\mathbf{1}} \cap \boldsymbol{S}_{\mathbf{2}} \cap \boldsymbol{S}_{\mathbf{3}}$. In practice, we have to check that the reconstructed signal $\boldsymbol{y}_{2}$ (see Fig. 1) is the result of filtering a periodic stream of Diracs. In terms of Fourier coefficients, Strong consistent reconstruction means that $\boldsymbol{y}_{2}$ has Fourier coefficients satisfying (1). Notice that although for high enough oversampling, the projection $\boldsymbol{P}_{\mathbf{3}}$ is convex, the set $\boldsymbol{S}_{\mathbf{3}}$ is not convex. In general, this could cause problems when iterating 


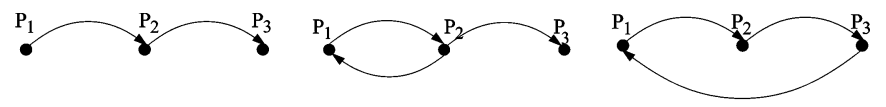

(a)

(b)

(c)

Fig. 4. (a) Nonconsistent reconstruction algorithm consists of applying projections $\boldsymbol{P}_{1}, \boldsymbol{P}_{\mathbf{2}}$ and $\boldsymbol{P}_{\mathbf{3}}$ only once. (b) The Weak consistency algorithm consists of first iterating projections $\boldsymbol{P}_{1}$ and $\boldsymbol{P}_{2}$, and then applying the projection $\boldsymbol{P}_{\mathbf{3}}$. (c) The Strong consistency algorithm consists of iterating the projections $\boldsymbol{P}_{1}, \boldsymbol{P}_{2}$, and $\boldsymbol{P}_{3}$.

the composite projection $\boldsymbol{P}_{\mathbf{3}} \boldsymbol{P}_{\mathbf{2}} \boldsymbol{P}_{\mathbf{1}}$, because while any projection $\boldsymbol{P}_{\boldsymbol{i}}$ mapping to $\boldsymbol{S}_{\boldsymbol{i}}$ will reduce (more precisely, not increase) the distance to $\boldsymbol{S}_{\boldsymbol{i}}$, if one of the sets is not convex, we could still get an increase in distance to the intersection $\boldsymbol{S}_{\mathbf{1}} \cap \boldsymbol{S}_{\mathbf{2}} \cap \boldsymbol{S}_{\mathbf{3}}$. Here, we conjecture that for large enough $R_{t}$ and $R_{f}$, the convergence property is ensured. Our experimental results in Section VI confirm clearly this conjecture. The Strong consistency algorithm is illustrated in Fig. 4(c). Notice that the complexity of the Strong consistent algorithm is higher than of the Weak consistent algorithm.

To illustrate the fact that Strong consistency introduces one more set of constraints and hence reduces the set of possible reconstructions, as compared to Weak consistency, we remark that

$S_{1} \cap S_{2} \cap S_{3} \subset S_{3} \Rightarrow P_{3}\left(S_{1} \cap S_{2} \cap S_{3}\right)=S_{1} \cap S_{2} \cap S_{3}$.

On the other hand

$$
P_{3}\left(S_{1} \cap S_{2} \cap S_{3}\right) \subset P_{3}\left(S_{1} \cap S_{2}\right)
$$

that confirms that the set of Strong consistent reconstruction is a subset of the Weak consistent reconstruction set. This implies that by enforcing Strong consistency, the reconstruction will be, on average, closer (or the same, but never further) to the original signal, than by enforcing only Weak consistency.

It is important to notice that, since $\boldsymbol{y}_{\mathbf{2}}$ is a bandlimited signal, there exist algorithms [16]-[18] for reconstructing $\boldsymbol{y}_{2}$ which do not require iterated projections and which achieve a similar reconstruction accuracy as the one shown in Theorem 1 (see Section V). These algorithms could then be followed by projection $\boldsymbol{P}_{\mathbf{3}}$ in order to achieve a performance similar to the Weak consistency algorithm. However, the algorithms in [16]-[18] do not ensure the consistency with respect to the quantization bins, i.e., set $\boldsymbol{S}_{\mathbf{1}}$, which means that they cannot be used, together with projection $\boldsymbol{P}_{\mathbf{3}}$, in order to achieve Strong consistency.

\section{A. Extension to Other Nonbandlimited Signals With Finite Rate of Innovations}

Our reconstruction algorithms can be applied to other types of signals with finite rate of innovation, such as finite (nonperiodic) streams of Diracs and periodic nonuniform splines, where oversampling in time and in frequency can be again introduced.

The reconstruction of finite streams of Diracs from filtered unquantized samples, is explained in [1]. Basically, after getting the filtered samples $y_{n}$, using a sinc sampling kernel, an annihilating discrete-time filter method is used to obtain, first, the time positions and then the weights. In the case of quantization, after quantizing the samples $y_{n}$, as before, we can project to the space of (nonperiodic) bandlimited signals, with bandwidth determined by the oversampling in frequency, and check if the new samples $\hat{y}_{n}$ belong to the corresponding quantization bins. If this is not the case, we can perform projection $\boldsymbol{P}_{\mathbf{1}}$, as before. Similarly to $\boldsymbol{P}_{\mathbf{3}}$, we can define a projection on the signal space of finite streams of Diracs.

Analyzing periodic nonuniform splines, we can see that the $(S+1)$ th derivative of a periodic nonuniform spline of degree $S$ with knots at $\left\{t_{k}\right\}_{k=0}^{K-1}$ is given by a periodic stream of $K$ Diracs. This allows us to extend easily the reconstruction algorithm to the case of nonuniform splines. The $(S+1)$ th derivative of a nonuniform spline $x^{(S+1)}(t)$ has Fourier coefficients given by

$$
X^{(S+1)}[m]=\frac{1}{\tau} \sum_{k=0}^{K-1} c_{k} e^{-j 2 \pi m t_{k}} .
$$

Differentiating (1) $S+1$ times we see that, the Fourier coefficients $X^{(S+1)}[m]$ are related to the Fourier coefficients $X[m]$ of $x(t)$ in the following way:

$$
X^{(S+1)}[m]=\left(\frac{j 2 \pi m}{\tau}\right)^{(S+1)} X[m], \quad m \in \mathcal{Z} .
$$

Therefore, we can use the same consistent reconstruction algorithms to obtain $X^{(S+1)}[m]$, providing the final reconstruction of the nonuniform splines.

\section{Theoretical Performance of Oversampling}

\section{A. Error in Time Positions $\left\{t_{k}\right\}$}

As explained earlier, in order to estimate the time positions and weights, some of the consistency constraints that we enforce involve the $N$-periodic bandlimited $(M \leq N)$ discrete-time signal $\boldsymbol{y}_{2}$. We can easily change the bandwidth of the signal $\boldsymbol{y}_{2}$, by increasing/decreasing the bandwidth of the sampling kernel $h_{B}$, which is equivalent to changing $R_{f}$. In terms of DTFS, increasing/decreasing the bandwidth is equivalent to adding/removing nonzero DTFS. Similarly, for the fixed bandwidth we still can choose how many samples $\boldsymbol{y}$ we want to have, or equivalently, choose $R_{t}$. In the following, we are going to see what is the dependence of the reconstruction quality on $R_{t}$ and $R_{f}$. As a quantitative characterization of the reconstruction quality we introduce the following distances:

1) $d_{1}(\boldsymbol{y}, \hat{\boldsymbol{y}})=\operatorname{MSE}(\boldsymbol{y}, \hat{\boldsymbol{y}})=\operatorname{MSE}(\boldsymbol{Y}, \hat{\boldsymbol{Y}})$ (Parseval's theorem);

2) $d_{2}(\boldsymbol{y}, \hat{\boldsymbol{y}})=\operatorname{MSE}\left(\boldsymbol{P}_{\mathbf{3}}\left(\boldsymbol{P}_{\mathbf{2}}(\boldsymbol{y})\right), \boldsymbol{P}_{\mathbf{3}}\left(\boldsymbol{P}_{\mathbf{2}}(\hat{\boldsymbol{y}})\right)\right)$;

3) $d_{3}(\boldsymbol{y}, \hat{\boldsymbol{y}})=\operatorname{MSE}(\boldsymbol{t}, \hat{\boldsymbol{t}})$;

4) $d_{4}(\boldsymbol{y}, \hat{\boldsymbol{y}})=\operatorname{MSE}(\boldsymbol{c}, \hat{\boldsymbol{c}})$.

In practice, the distances $d_{2}, d_{3}$ and $d_{4}$ are the most interesting. However, in the following two theorems we speak about $d_{1}$ and $d_{3}$. This is because, as an intermediate step to compute $d_{3}$ we need to make use of the distance $d_{1}$ for the case of $\boldsymbol{y} \in \boldsymbol{S}_{\mathbf{1}} \cap \boldsymbol{S}_{\mathbf{2}}$. Later, we also show theoretically that $d_{4}$ depends on $d_{3}$ and experimentally that $d_{1}$ does not differ too much from $d_{2}$ when $\boldsymbol{y} \in \boldsymbol{S}_{\mathbf{1}} \cap \boldsymbol{S}_{\mathbf{2}}$ (see Fig. 5). For the Strong consistent reconstruction the distances $d_{2}$ and $d_{3}$, when $\boldsymbol{y} \in \boldsymbol{S}_{\mathbf{1}} \cap \boldsymbol{S}_{\mathbf{2}} \cap \boldsymbol{S}_{\mathbf{3}}$, are experimentally shown later.

Theorem 1: Given the two $N$-periodic discrete time signals $\boldsymbol{y}, \hat{\boldsymbol{y}} \in \boldsymbol{S}_{\mathbf{1}} \cap \boldsymbol{S}_{\mathbf{2}}$ where the sets $\boldsymbol{S}_{\mathbf{1}}$ and $\boldsymbol{S}_{\mathbf{2}}$ are uniquely determined by $x(t), R_{t}$ and $R_{f}$, there exists an $N_{0}$ such that if 


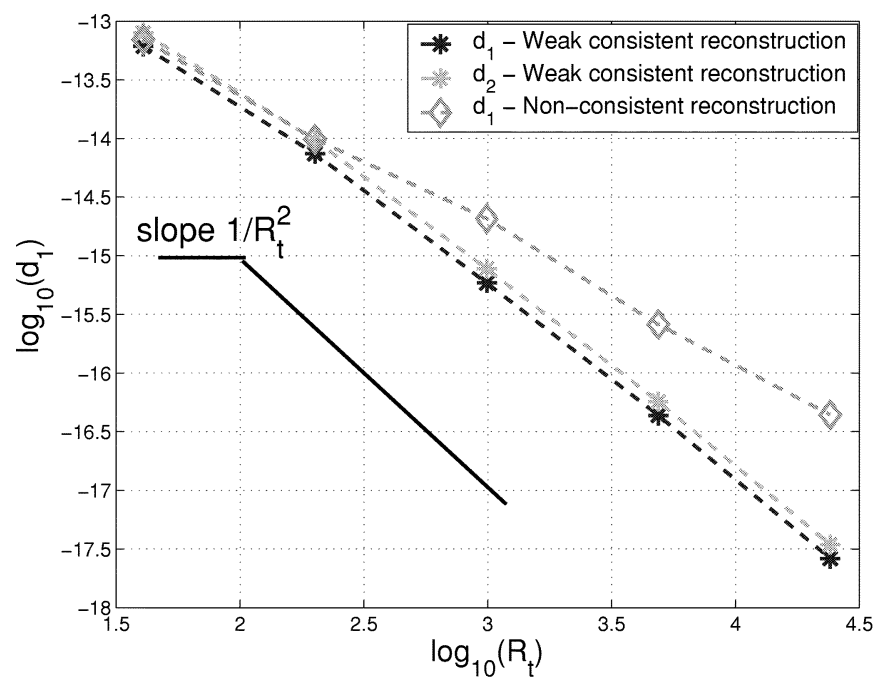

(a)

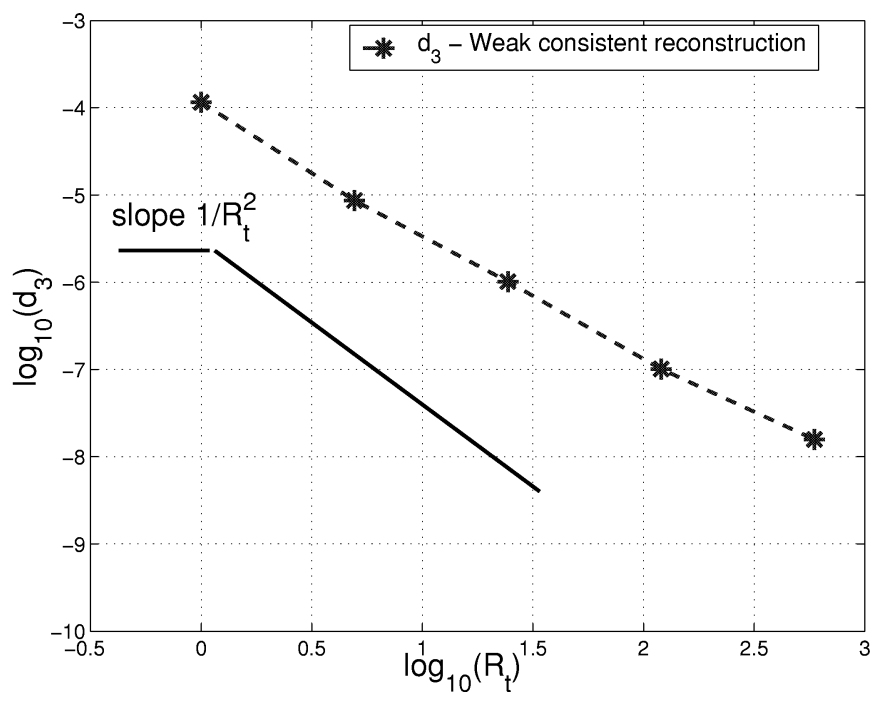

(b)

Fig. 5. The Weak consistent reconstruction. Dependence of accuracy on oversampling in time $R_{t}$ for (a) $d_{1}-\mathrm{MSE}$ of Fourier coefficients where $X[m]=Y_{2}[m]$ and $Y_{2}^{\prime}[m]$ is a reconstruction. (b) $d_{3}-\mathrm{MSE}$ of time positions.

$N \geq N_{0}$, there is a constant $c>0$, which depends only on $x(t)$ and not on $R_{t}$ and $R_{f}$, such that

$$
d_{1}(\boldsymbol{y}, \hat{\boldsymbol{y}}) \leq \frac{c}{R_{t}^{2}} .
$$

Proof: See Appendix I.

The importance of this theorem is in the fact that even if we increase $R_{f}$, while keeping $R_{t}$ constant, the upper bound on $d_{1}$ remains the same. However, it is clear that since, we estimate the time positions from DTFS of $\boldsymbol{y}_{2}$, the number of available Fourier components in addition to $d_{1}$ directly impacts $d_{3}$. That is, increasing $R_{f}$, intuitively, will improve the time positions estimates. On the other hand, notice also that, since from Theorem $1 d_{1}$ will decrease as we increase $R_{t}$, then $d_{3}$ will decrease as we increase both $R_{t}$ and $R_{f}$. Here, we also remark that increasing $R_{t}$ and/or $R_{f}$, we also increase the number of samples since $N=(2 K+1) R_{t} R_{f}$.

In the following theorem, we examine the order of $d_{3}$ as a function of both oversamplings $R_{t}$ and $R_{f}$, for the case of Weak consistent reconstruction.

Theorem 2: Given the two $N$-periodic discrete time signals $\boldsymbol{y}, \hat{\boldsymbol{y}} \in \boldsymbol{S}_{\mathbf{1}} \cap \boldsymbol{S}_{\mathbf{2}}$ where the sets $\boldsymbol{S}_{\mathbf{1}}$ and $\boldsymbol{S}_{\mathbf{2}}$ are uniquely determined by $x(t), R_{t}$ and $R_{f}$, there exist some constants $a \geq 1$ and $b \geq 1$, such that if $R_{t} \geq a$ and $R_{f} \geq b$, there is a constant $c^{\prime}>0$ which depends only on $x(t)$ and not on $R_{t}$ and $R_{f}$, and

$$
d_{3}(\boldsymbol{y}, \hat{\boldsymbol{y}}) \leq \frac{c^{\prime}}{R_{f}^{3} R_{t}^{2}} .
$$

Proof: See Appendix II.

From Theorem 2 it can be seen that if we are limited to some large but finite number of samples and $N \gg 2 K+1$, by increasing $R_{f}$ we reduce $d_{3}$ faster than by increasing $R_{t}$. Thus, if we are allowed to use a fixed number of samples $N$ and our goal is to minimize only $d_{3}$ we will tend to increase oversampling in frequency, rather than oversampling in time. In Sections VII and VIII the influence of increasing $R_{t}$ and $R_{f}$ on the required bit-rate and error-rate dependence will be considered, as well.

\section{B. Error in Weights $\left\{c_{k}\right\}$}

Given the time position estimates $\left\{\hat{t}_{k}\right\}$ we can directly estimate the weights $\left\{\hat{c}_{k}\right\}$ from (1) as

$$
\left(\begin{array}{c}
X[0] \\
X[1] \\
\vdots \\
X[M]
\end{array}\right)=\frac{1}{\tau}\left(\begin{array}{cccc}
1 & 1 & \cdots & 1 \\
u_{0} & u_{1} & \cdots & u_{K-1} \\
\vdots & \vdots & \cdots & \vdots \\
u_{0}^{M} & u_{1}^{M} & \cdots & u_{K-1}^{M}
\end{array}\right)\left(\begin{array}{c}
c_{0} \\
c_{1} \\
\vdots \\
c_{K-1}
\end{array}\right) .
$$

Notice here, that the Fourier coefficients are coming as the result of the projection $\boldsymbol{P}_{2}$, that is $\boldsymbol{X}=\boldsymbol{Y}_{\mathbf{2}}$. We can also write the previous equation in the matrix notation as follows:

$$
Y_{2}=\frac{1}{\tau} \boldsymbol{V} C
$$

Notice that the matrix $\boldsymbol{V}$ has $M \geq K$ rows, due to the oversampling in time, which implies that the system in (7) is overdetermined. Thus, we can compute $\boldsymbol{C}$ in the two ways, using a TLS projection or a LS projection. In the following, $\boldsymbol{C}$ is given by

$$
\boldsymbol{C}=\left(\boldsymbol{V}^{H} \boldsymbol{V}\right)^{-1} \boldsymbol{V}^{H} \boldsymbol{Y}_{2} .
$$

The error in vector $C$ defined by $d_{4}$ depends directly on the error in $Y_{2}$, which is equal to $d_{1}$ in the case of Weak consistent reconstruction, and on the error in $\boldsymbol{V}$ which is related to the error in time positions $d_{3}$. Hence

$$
d_{4}(\boldsymbol{y}, \hat{\boldsymbol{y}})=f\left(d_{1}(\boldsymbol{y}, \hat{\boldsymbol{y}}), d_{3}(\boldsymbol{y}, \hat{\boldsymbol{y}})\right) \quad \text { for } \quad \boldsymbol{y}, \hat{\boldsymbol{y}} \in \boldsymbol{S}_{\mathbf{1}} \cap \boldsymbol{S}_{\mathbf{2}} .
$$

Because of this dependence, in this paper, we focus on the error $d_{1}$ and $d_{3}$. Moreover, in many practical applications, such as UWB communications (e.g., PPM modulation) and sensor networks sampling local physical sources, the important information is given by time positions.

\section{EXPERIMENTAL PERFoRMANCE OF OVERSAMPLING}

In this section, we show experimental results for the three algorithms illustrated in Fig. 4, with parameters: $K=2, \tau=10$, $t_{k} \in(0, \tau], c_{k} \in[-1,1]$. The positions and the weights are 


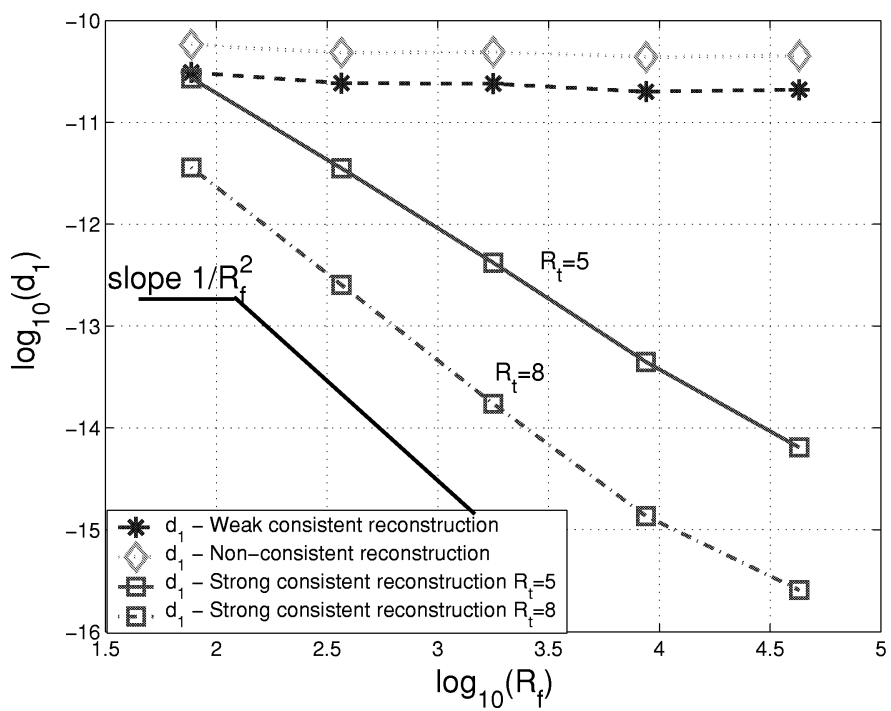

(a)

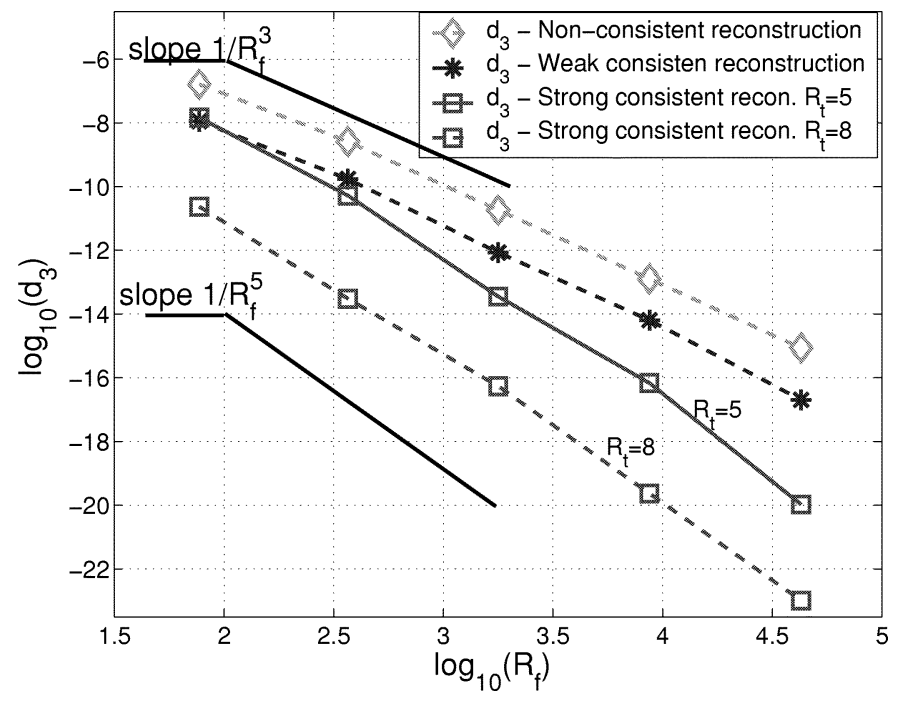

(b)

Fig. 6. The nonconsistent, Weak consistent and Strong consistent reconstruction. Dependence of accuracy on oversampling in frequency $R_{f}$ for (a) $d_{1}-\mathrm{MSE}$ of Fourier coefficients where $X[m]=Y_{2}[m]$ and $Y_{2}^{\prime}[\mathrm{m}]$ is a reconstruction. (b) $d_{3}-$ MSE of time positions.

randomly chosen from the corresponding intervals and the results are the average over 300 signals. For the Weak consistency algorithm, our numerical results illustrated in Fig. 5 and Fig. 6 confirm Theorems 1 and Theorem 2. The Strong consistency algorithm provides an experimental behavior of $O\left(1 / R_{t}^{2} R_{f}^{5}\right)$ that is also illustrated in Fig. 6. We have also compared our consistent reconstruction algorithms with the case of nonconsistent reconstruction. A clear outperformance of our reconstruction algorithms over nonconsistent reconstructions is observed [see Figs. 5(a) and 6].

We can conclude that by increasing the oversampling in frequency $R_{f}$, we can achieve a reconstruction accuracy which is (polynomially) superior for both the Weak and the Strong consistency algorithms than the one obtained by increasing $R_{t}$. Moreover, from the results of MSE dependence on the quantization stepsize derived in Section VIII, we also conclude that oversampling in frequency outperforms decreasing of the quantization stepsize $\Delta$. Therefore, oversampling in frequency provides largest gain in performance.

\section{ENCODING SCHEME AND BIT RATE}

In this section, we analyze encoding schemes and the scaling laws that can be achieved in terms of bit rate and error-rate dependence. As explained in Section III, by increasing $R_{t}$ or $R_{f}$, we increase the number of samples $\left\{y_{n}\right\}$. It is clear that using the traditional way of encoding, that is, pulse-code modulation (PCM) encoding, the bit rate depends linearly on the number of samples $N$. For each sample, using a scalar quantizer with stepsize $\Delta$, we need at most $1+\log _{2}\left(d_{y} / \Delta\right)$ bits, where $d_{y}$ denotes the dynamic range in amplitude of the signal $y(t)$. From (15) it can be easily shown that

$$
d_{y} \leq 2\left(\sum_{k=0}^{K}\left|c_{k}\right|\right) \frac{2 M+1}{\tau} \Longrightarrow d_{y}=O\left(R_{f}\right) .
$$

In the previous equation, we assume that the weights are bounded by some fixed bounds, that is, $c_{k} \in[-a, a]$. Hence, for a fixed $\Delta$, the bit rate can be bounded as

$$
\begin{aligned}
B_{P C M} & =\frac{N}{\tau}\left(1+\log _{2}\left(\frac{d_{y}}{\Delta}\right)\right) \\
& =N O\left(\log _{2}\left(R_{f}\right)\right) \\
& =O\left(R_{t} R_{f} \log _{2} R_{f}\right) .
\end{aligned}
$$

On the other hand, when the sampling interval is sufficiently fine, some simple and efficient techniques can be developed [10], for which the required bit rate is substantially smaller than in the case of PCM encoding. In the following, we show we can use the results in [10], developed for bandlimited signals to compute the dependence of the bit rate on both $R_{t}$ and $R_{f}$.

The idea originates from the equivalence between the traditional interpretation of the digital version of an analog signal, where the uncertainty is determined by the quantization stepsize at the exact time instants, and the alternative one [10], [19], where the digital signal is uniquely determined by the sampling intervals in which its quantization threshold crossings occur. A unique representation in the alternative interpretation is ensured if the following two conditions are satisfied: 1) the quantization threshold crossings are sufficiently separated; 2) at most one quantization threshold crossing occurs in each sampling interval. The first condition requires that the intervals between consecutive crossings of any given threshold are limited from below by a constant $T_{1}>0$. The second condition is satisfied if the slope of the signal is finite. The latter is ensured by the fact that the signal $y(t)$ has finite energy and is bandlimited. Thus, there is always an interval $T_{2}>0$ on which $y(t)$ cannot go through more that one quantization threshold crossing. For a sufficiently fine sampling period, that is $T_{s} \leq \min \left(T_{1}, T_{2}\right)$, all quantization threshold crossings occur in distinct sampling intervals, and a unique representation is ensured.

The encoded information, in the case of threshold crossings (TC) based encoding, are the positions of the sampling intervals in which the quantization threshold crossings occur. The 
signal is observed in a given time interval, which in our case is the period $\tau$. For determining the position of each sampling interval of length $T_{s}=\tau / N$, we need at most $1+\log _{2}\left(\tau / T_{s}\right)$ bits. Every threshold crossing can be determined with respect to the previous one by introducing only one additional bit to indicate the direction, upwards or downwards, of the next threshold crossing. If $C$ quantization threshold crossings occur during the period $\tau$, then the required bit rate is

$$
B_{T C}=C\left(2+\log _{2}\left(T_{s} / \tau\right)\right) .
$$

Next, we need to determine the maximal number of threshold crossings. There are two types of threshold crossings: 1) a d-crossing which is preceded by a threshold crossing of a different threshold level [Fig. 3(a)-the first and the second threshold crossing], and 2) an s-crossing which is preceded by a crossing of the same threshold level [Fig. 3(a)-second and third threshold crossing]. The sum of these two types of threshold crossings is the total number of threshold crossings. From results on nonharmonic Fourier expansions [20], the s-crossings for the case of bandlimited signals constitute a sequence of uniform density ${ }^{2} \rho$. This also means that the zeros of the first derivative of $y(t)$ constitute a sequence of the same density. Then, $\rho$ is bounded as $\rho=O\left(f_{\max }\right)=O\left(R_{f}\right)$, where $f_{\max }$ is the maximum frequency of the signal $y(t)$ and it is of the order of the bandwidth $B$. Consequently, the number of s-crossings $C_{s}$ is given by $C_{s}=O\left(f_{\max }\right)=O\left(R_{f}\right)$. The maximum possible number of d-crossings $C_{d}$ depends linearly on the maximum dynamic range $d_{y}$ of the signal $y(t)$. Using (8) we have that $C_{d}=O\left(d_{y} / \Delta\right)=O\left(R_{f}\right)$. Therefore

$$
B_{T C} \leq c_{3} R_{f}\left(2+\log _{2}\left(c_{4} R_{t} R_{f}\right)\right)
$$

where $c_{3}$ and $c_{4}$ are some constants that depend on the specific signal $x(t)$ and on the quantization stepsize $\Delta$, but which do not depend on $R_{t}$ and $R_{f}$. The additional bits required for specifying the first threshold crossing (the others are going to be specified with respect to this one) have arbitrary small effect on the required bit rate over the sufficiently long time period.

Comparing (9) and (10), we can conclude that the TC based encoding has clear advantages over the traditional PCM encoding, since the bit-rate for TC based encoding grows much more slowly as a function of the oversampling in time $R_{t}$. We also remark that these coding results are applicable regardless of the reconstruction method that is used (e.g., consistent or nonconsistent reconstruction).

\section{ERROR-RATE DEPENDENCE}

A natural question that arises in oversampled A/D conversion is to compare the improvement in error-rate that comes, on the one hand, from the oversamplings, in our case from increasing $R_{t}$ and $R_{f}$, and on the other hand, from reducing the quantization stepsize $\Delta$. To represent the error-rate dependence, we consider the distance $d_{3}$ as a function of the bit rate.

\footnotetext{
${ }^{2} \mathrm{~A}$ sequence $\lambda_{n}$ of real or complex numbers has uniform density $\rho, \rho \geq 0$, if there are constants $L \leq \infty$ and $s>0$ such that: a) $\left|\lambda_{n}-(n / \rho)\right| \leq L, n \in \mathbb{Z}$, b) $\left|\lambda_{n}-\lambda_{m}\right| \geq s>0$, where $n \neq m$.
}

We have shown in Section V and Section VII the behavior of both $d_{3}$ and the bit rate as a function of $R_{t}$ and $R_{f}$. In this section, we also introduce the corresponding behavior as a function of the quantization stepsize $\Delta$, and then we examine the error-rate dependence considering all the three parameters, $R_{t}$, $R_{f}$, and $\Delta$.

It has been shown in [10] that if: 1) there is a large number of quantization levels compared to the signal amplitude range, and 2 ) the quantization stepsize $\Delta$ is sufficiently small, then, it is approximately correct to model the quantization error as a uniformly distributed white noise over the interval $[-\Delta / 2, \Delta / 2]$ that is independent of the input signal. Assuming the white noise based model, we have

$$
d_{1}(\boldsymbol{y}, \hat{\boldsymbol{y}})=\operatorname{MSE}\left(\boldsymbol{Y}_{\mathbf{2}}, \hat{\boldsymbol{Y}}_{\mathbf{2}}\right)=\frac{\Delta^{2}}{12} .
$$

Recall from Section II that we estimate the time positions from the Fourier coefficients $X[m]=Y_{2}[m]$, hence, the error $\mathcal{E}\left(t_{k}\right)$ for the time positions can be computed to a first order approximation $^{3}$ as:

$$
\begin{aligned}
\mathcal{E}\left(t_{k}\right) & =\sum_{m=-M}^{M} \frac{\partial t_{k}}{\partial Y_{2}[m]} \mathcal{E}\left(Y_{2}[m]\right) \\
\left|\mathcal{E}\left(t_{k}\right)\right|^{2} & \leq\left(\sum_{m=-M}^{M}\left|\frac{\partial t_{k}}{\partial Y_{2}[m]}\right|^{2}\right)\left(\sum_{m=-M}^{M}\left|\mathcal{E}\left(Y_{2}[m]\right)\right|^{2}\right) \\
& =\xi d_{1}
\end{aligned}
$$

where $\xi=\sum_{m=-M}^{M}\left|\left(\partial t_{k} / \partial Y_{2}[m]\right)\right|^{2}$ measures the dependence of the time positions on the Fourier coefficients and does not depend on the quantization stepsize $\Delta$. The inequality in (11) follows simply from the Cauchy-Schwartz inequality. Therefore, for sufficiently small $\Delta$ it holds that

$$
d_{3}(\boldsymbol{y}, \hat{\boldsymbol{y}})=O\left(\Delta^{2}\right) \text {. }
$$

Concerning the bit rate, it is clear that increasing/decreasing the quantization stepsize, we reduce/increase the required bit rate.

In the case of PCM encoding, for a fixed $R_{t}$ and $R_{f}$, it can be seen from (9) that, the bit rate, as a function of $\Delta$, is given by

$$
B_{P C M}=N\left(1+\log _{2}\left(\frac{d_{y}}{\Delta}\right)\right)=O\left(\log _{2}\left(\frac{1}{\Delta}\right)\right) .
$$

In the case of TC based encoding, the dependence of the bitrate on the quantization step size $\Delta$ comes through the dependence on the maximum number of threshold crossings, namely, $C_{\text {max }}(\Delta)=O(1 / \Delta)$, which together with (10) and taking into account that now $R_{t}$ and $R_{f}$ are fixed, results in

$$
B_{T C} \leq c_{5} \frac{1}{\Delta} R_{f}\left(2+\log _{2}\left(c_{4} R_{t} R_{f}\right)\right)=O\left(\frac{1}{\Delta}\right)
$$

where $c_{5}$ is some constant that does not depend on $R_{t}, R_{f}$ and $\Delta$. All the results, for the $d_{3}(\boldsymbol{y}, \hat{\boldsymbol{y}})$ and the bit rate, are given in Table I.

Analyzing Table I, we can see first that in the case of PCM encoding, the best error-rate dependence is obtained by decreasing the quantization stepsize $\Delta$ instead of increasing any type of oversampling. Since we have a logarithmic increase

\footnotetext{
${ }^{3}$ We assume sufficiently high enough oversamplings and sufficiently small
} quantization stepsize, so that the first order approximation is correct. 
TABLE I

ERROR-RATE DEPENDENCE

\begin{tabular}{|c||c||c||cc|cc|}
\hline Encoding method & Variation & Bit rate & Weak consistency - theory & Strong consistency - experiments \\
\cline { 3 - 6 } & & & $d_{3}(\boldsymbol{y}, \hat{\boldsymbol{y}})$ & error-rate & $d_{3}(\boldsymbol{y}, \hat{\boldsymbol{y}})$ & error-rate \\
\hline \multirow{3}{*}{ Threshold crossing encoding } & $R_{t}$ & $O\left(\log _{2} R_{t}\right)$ & $O\left(1 / R_{t}^{2}\right)$ & $\boldsymbol{O}\left(\mathbf{2}^{\left.-\mathbf{2} \boldsymbol{\alpha} \boldsymbol{B}_{T C}\right)}\right.$ & $O\left(1 / R_{t}^{2}\right)$ & $\boldsymbol{O}\left(\mathbf{2}^{\left.-\mathbf{2} \boldsymbol{\alpha} \boldsymbol{B}_{T C}\right)}\right.$ \\
& $R_{f}$ & $O\left(R_{f} \log _{2} R_{f}\right)$ & $O\left(1 / R_{f}^{3}\right)$ & $O\left(B_{T C}^{-3}\right)$ & $O\left(1 / R_{f}^{5}\right)$ & $O\left(B_{T C}^{-5}\right)$ \\
& $\Delta$ & $O(1 / \Delta)$ & $O\left(\Delta^{2}\right)$ & $O\left(B_{T C}^{-2}\right)$ & $O\left(\Delta^{2}\right)$ & $O\left(B_{T C}^{-2}\right)$ \\
\hline \multirow{3}{*}{ PCM encoding } & $R_{t}$ & $O\left(R_{t}\right)$ & $O\left(1 / R_{t}^{2}\right)$ & $O\left(B_{P C M}^{-2}\right)$ & $O\left(1 / R_{t}^{2}\right)$ & $O\left(B_{P C M}^{-2}\right)$ \\
& $R_{f}$ & $O\left(R_{f} \log _{2} R_{f}\right)$ & $O\left(1 / R_{f}^{3}\right)$ & $O\left(B_{P C M}^{-3}\right)$ & $O\left(1 / R_{f}^{5}\right)$ & $O\left(B_{P C M}^{-5}\right)$ \\
& $\Delta$ & $O\left(\log _{2}(1 / \Delta)\right)$ & $O\left(\Delta^{2}\right)$ & $\boldsymbol{O}\left(\mathbf{2}^{-2} \boldsymbol{\beta} B_{P C M}\right)$ & $O\left(\Delta^{2}\right)$ & $\boldsymbol{O}\left(\mathbf{2}^{-\mathbf{2} \boldsymbol{\beta} B P C M}\right)$ \\
\hline
\end{tabular}

$O\left(\log _{2}(1 / \Delta)\right)$ of the bit rate and an error decrease of $O\left(\Delta^{2}\right)$, changing the stepsize $\Delta$ and fixing the oversampling ratios, we get a dependence for $d_{3}$ of $O\left(2^{-2 \beta B_{\mathrm{PCM}}}\right)$, where $\beta$ is some constant that does not depend on $B_{\mathrm{PCM}}$. Assuming PCM encoding, this performance can not be achieved by increasing the oversampling ratios $R_{t}$ and $R_{f}$. However, using the TC based encoding, we can achieve the same dependence (as when reducing the quantization stepsize $\Delta$ ), by increasing the oversampling in time $R_{t}$. This is very important because, in practice, the cost (complexity of expensive high-precision analog circuitry) of halving $\Delta$ is much higher than that of doubling $R_{t}$. However, by increasing the oversampling in frequency $R_{f}$, the required bit rate grows exponentially faster than in the case of increasing $R_{t}$ or decreasing $\Delta$. Therefore, asymptotically (high rates), the error-rate performance obtained by increasing $R_{f}$ is inferior to that of increasing $R_{t}$ or decreasing $\Delta$.

\section{CONCLUSION AND Future WORK}

In this paper, we studied reconstruction of nonbandlimited signals with finite rate of innovation, particularly, periodic streams of Diracs, under the presence of quantization. High reconstruction accuracy is obtained by introducing the oversampling in time and in frequency, and enforcing the concept of consistency. We defined the concept of Weak and Strong consistency and we examined the performance in terms of MSE of time positions, that is achieved with Weak and Strong consistent reconstruction algorithms. We concluded that the oversampling in frequency provides a superior decrease in MSE of time positions. On the other hand, in terms of error-rate dependence, by using a threshold crossing based encoding, the oversampling in time provides a superior error-rate tradeoff over the oversampling in frequency. Moreover, it is also observed that the error-rate dependence obtained from doubling the oversampling in time is the same as the one obtained from halving the quantization stepsize, while, in practice, the cost of performing the oversampling in time is much lower than that of reducing the quantization stepsize.

Some future lines of research include finding faster consistent reconstruction algorithms, such as algorithms which do not require projection iterations, extension of our results of $\mathrm{A} / \mathrm{D}$ conversion for the case of more general sampling kernels (e.g., Gaussian kernels), reconstruction of signals with finite rate of innovations under physics based kernels, such as those given by a heat diffusion equation [5], and extension of our results to multidimensional nonbandlimited signals with finite rate of innovations.

\section{APPENDIX I \\ PROOF OF THEOREM 1}

In order to prove Theorem 1, we first need to compute the slope of the filtered signal $y(t)$. This signal is nothing but the sum of " $2 M+1$-periodized" sinc functions, that is

$$
y(t)=\sum_{k=0}^{K} c_{k} \frac{\sin \left((2 M+1)\left(t-t_{k}\right) \pi / \tau\right)}{\sin \left(\left(t-t_{k}\right) \pi / \tau\right)} .
$$

It is then obvious that the slope of $y(t)$ is of the following order

$$
\frac{d y(t)}{d t}= \begin{cases}O(1), & \text { for } t=t_{k} \\ O\left(R_{f}\right) & \text { otherwise. }\end{cases}
$$

Using the results on oversampled $A / D$ conversion of bandlimited signals in $L^{2}(\mathbb{R})$ [10], it can be shown that in the case of stable sampling ${ }^{4}$ [21], which is satisfied by the class of periodic bandlimited signals, the $\operatorname{MSE}(y(t), \hat{y}(t))$ can be written as follows:

$$
\operatorname{MSE}(y(t), \hat{y}(t)) \leq \frac{9}{4} \frac{B}{A} \frac{\tau^{2}}{N^{2}}\left\|y^{\prime}(t)\right\|^{2}
$$

where $A>0$ and $B<\infty$ are some constants that do not depend on the signal $y(t)$ (particularly, they come from the definition of stable sampling) and $\|\cdot\|$ is the $L^{2}(\mathbb{R})$ norm. Inserting (16) in (17), we get the final conclusion

$$
d_{1}(\boldsymbol{y}, \hat{\boldsymbol{y}}) \leq \frac{9}{4} \frac{B}{A} \frac{\tau^{2}}{(2 K+1)^{2} R_{t}^{2} R_{f}^{2}} O\left(R_{f}^{2}\right)=O\left(\frac{1}{R_{t}^{2}}\right) .
$$

Here, $\boldsymbol{y}$ and $\hat{\boldsymbol{y}}$ are any two signals in $\boldsymbol{S}_{\mathbf{1}} \cap \boldsymbol{S}_{\mathbf{2}}$.

\section{APPENDIX II \\ PROOF OF THEOREM 2}

The annihilating filter method with oversampling in time and in frequency is the classical high-order Yule-Walker system (HOYW) [12]. In order to prove Theorem 2, we go through the two main steps:

${ }^{4}$ Definition of stable sampling: A sequence of real numbers $\left(\lambda_{n}\right)_{n \in \mathcal{Z}}$ is said to be a sequence of stable sampling in the space of square-integrable $\pi$-bandlimited function, denoted by $\mathcal{V}_{\pi}$, if there exist two constants, $A>0$ and $B<\infty$, such that for any $f$ in $\mathcal{V}_{\pi}$, the following sequence holds:

$$
A \int_{-\infty}^{\infty}|f(x)|^{2} d x \leq \sum_{n} \mid f\left(\left.\lambda_{n}\right|^{2} \leq B \int_{-\infty}^{\infty}|f(x)|^{2} d x\right.
$$


1) We first show how the estimation accuracy is effected by a) the number of YW equations, or equivalently, the oversampling in frequency $R_{f}$;

b) the model order, or equivalently, the filter order L.

For this purpose, we use a common singular value decomposition (SVD)-based HOYW procedure.

2) Then, we use the known result that the TLS-based HOYW method and the SVD-based HOYW method are asymptotically equivalent.

SVD-based method finds the rank- $K$ ( $K$ being the number of Diracs) best approximation $\hat{\boldsymbol{H}}_{K}$ in the Frobenious norm sense and obtains the following solution

$$
\begin{aligned}
\hat{\boldsymbol{H}}_{K} \hat{\boldsymbol{a}} & =-\hat{\boldsymbol{h}} \\
\hat{\boldsymbol{a}} & =-\left(\hat{\boldsymbol{H}}_{K}\right)^{\dagger} \hat{\boldsymbol{h}}
\end{aligned}
$$

where $(\cdot)^{\dagger}$ denotes the Moore-Penrose pseudoinverse of $(\cdot)$. In general, the matrix $\hat{\boldsymbol{H}}$ has full rank being equal to $\min (L, M)$ and $(\hat{\boldsymbol{H}})^{\dagger}$ does not approach $(\boldsymbol{H})^{\dagger}$ which has rank $K$, even for the case when $M$ increases without bound. In contrast to $(\hat{\boldsymbol{H}})^{\dagger}$, the pseudoinverse $\left(\hat{\boldsymbol{H}}_{K}\right)^{\dagger}$ tends to $(\boldsymbol{H})^{\dagger}$ as the error in $\hat{X}[m]$ becomes smaller. This is a crucial property that is necessary for reducing the error in the estimates.

Suppose that $\boldsymbol{a}$ are the exact coefficients of the annihilating filter polynomial. Then, the following analysis holds:

$$
\begin{aligned}
\hat{\boldsymbol{a}}-\boldsymbol{a} & =-\left(\hat{\boldsymbol{H}}_{K}\right)^{\dagger} \hat{\boldsymbol{h}}-\boldsymbol{a} \\
& =-\left(\hat{\boldsymbol{H}}_{K}\right)^{\dagger}(\hat{\boldsymbol{h}}+\hat{\boldsymbol{H}} \boldsymbol{a}-\hat{\boldsymbol{H}} \boldsymbol{a})-\boldsymbol{a} \\
& =-\left(\hat{\boldsymbol{H}}_{K}\right)^{\dagger}(\hat{\boldsymbol{h}}+\hat{\boldsymbol{H}} \boldsymbol{a})+\left[\left(\hat{\boldsymbol{H}}_{K}\right)^{\dagger} \hat{\boldsymbol{H}}-\boldsymbol{I}\right] \boldsymbol{a} \\
& =-\left(\hat{\boldsymbol{H}}_{K}\right)^{\dagger} \boldsymbol{\xi}+\left[\left(\hat{\boldsymbol{H}}_{K}\right)^{\dagger} \hat{\boldsymbol{H}}-\boldsymbol{I}\right] \boldsymbol{a}
\end{aligned}
$$

where

$$
\boldsymbol{\xi}=\hat{\boldsymbol{h}}+\hat{\boldsymbol{H}} \boldsymbol{a}=\left[\begin{array}{ll}
\hat{\boldsymbol{h}} & \hat{\boldsymbol{H}}
\end{array}\right]\left[\begin{array}{l}
1 \\
\boldsymbol{a}
\end{array}\right] .
$$

First, we show what is the relationship between the matrix $\mathcal{P}_{\boldsymbol{a}}=(\hat{\boldsymbol{a}}-\boldsymbol{a})(\hat{\boldsymbol{a}}-\boldsymbol{a})^{T}$, associated to the annihilating filter coefficients and the matrix $\mathcal{P}_{\boldsymbol{t}}=(\hat{\boldsymbol{t}}-\boldsymbol{t})(\hat{\boldsymbol{t}}-\boldsymbol{t})^{T}$, associated to the time positions.

The angular positions of the roots of the annihilating filter give us the estimates of the time positions. Assuming that the roots are sufficiently close to the unit circle, so that the first approximation of the Taylor series expansion corresponding to the time positions holds, it can be shown [12] that:

$$
\hat{t}_{k}-t_{k}=\frac{1}{2 \pi} \operatorname{Re}\left\{\frac{\beta_{k}-j \alpha_{k}}{\alpha_{k}^{2}+\beta_{k}^{2}} \boldsymbol{u}_{\boldsymbol{k}}^{T}(\hat{\boldsymbol{a}}-\boldsymbol{a})\right\}
$$

with $u_{k}=\left[u_{k}^{-1} u_{k}^{-2} \ldots u_{k}^{-L}\right]^{T}$ or, in matrix form,

$$
\hat{\boldsymbol{t}}-\boldsymbol{t}=\frac{1}{2 \pi} \operatorname{Re}\{\boldsymbol{F} G(\hat{\boldsymbol{a}}-\boldsymbol{a})\}
$$

Therefore

$$
\begin{aligned}
\mathcal{P}_{\boldsymbol{t}} & =(\hat{\boldsymbol{t}}-\boldsymbol{t})(\hat{\boldsymbol{t}}-\boldsymbol{t})^{T} \\
& =\frac{1}{4 \pi^{2}} \operatorname{Re}\{\boldsymbol{F} \boldsymbol{G}(\hat{\boldsymbol{a}}-\boldsymbol{a})\} \operatorname{Re}\left\{(\hat{\boldsymbol{a}}-\boldsymbol{a})^{T} \boldsymbol{G}^{T} \boldsymbol{F}^{T}\right\},
\end{aligned}
$$

where

$$
\begin{aligned}
& \alpha_{k}=\left[\begin{array}{llll}
\cos \left(2 \pi t_{k}\right) & 2 \cos \left(4 \pi t_{k}\right) & \ldots & L \cos \left(2 L \pi t_{k}\right)
\end{array}\right] \boldsymbol{a} \\
& \beta_{k}=\left[\begin{array}{llll}
\sin \left(2 \pi t_{k}\right) & 2 \sin \left(4 \pi t_{k}\right) & \ldots & L \sin \left(2 L \pi t_{k}\right)
\end{array}\right] \boldsymbol{a} \\
& \boldsymbol{F}=\left(\begin{array}{ccc}
\frac{\beta_{1}-j \alpha_{1}}{\alpha_{1}^{2}+\beta_{1}^{2}} & & 0 \\
& \ddots & \\
0 & & \frac{\beta_{K}-j \alpha_{K}}{\alpha_{K}^{2}+\beta_{K}^{2}}
\end{array}\right) \\
& \boldsymbol{G}=\left(\begin{array}{ccc}
u_{0}^{-1} & \ldots & u_{0}^{-L} \\
\vdots & & \vdots \\
u_{K-1}^{-1} & \ldots & u_{K-1}^{-L}
\end{array}\right) \text {. }
\end{aligned}
$$

Applying the same arguments as in [22], where a similar expression is found for the complex sinusoids in noise, it can be shown that

$$
\begin{aligned}
\mathcal{P}_{\boldsymbol{t}} & =\frac{1}{4 \pi^{2}} \operatorname{Re}\{\boldsymbol{F} \boldsymbol{G}(\hat{\boldsymbol{a}}-\boldsymbol{a})\} \operatorname{Re}\left\{(\hat{\boldsymbol{a}}-\boldsymbol{a})^{T} \boldsymbol{G}^{T} \boldsymbol{F}^{T}\right\} \\
& =\frac{1}{2} \frac{1}{4 \pi^{2}} \operatorname{Re}\left\{\boldsymbol{F} \boldsymbol{G} \mathcal{P}_{\boldsymbol{a}} \boldsymbol{G}^{H} \boldsymbol{F}^{H}\right\}
\end{aligned}
$$

where $(\cdot)^{H}$ denotes Hermitian transpose.

Unfortunately, it appears difficult to derive an explicit expression for the matrix $\mathcal{P}_{\boldsymbol{a}}$ for the various choices of $L$, because the vector $\boldsymbol{a}$ changes itself with $L$. However, it is possible to obtain a compact formula for $\mathcal{P}_{t}$ that is

$$
\mathcal{P}_{\boldsymbol{t}}=\frac{1}{2} \frac{1}{4 \pi^{2}} \operatorname{Re}\left\{\boldsymbol{F} \boldsymbol{G} \boldsymbol{H}^{\dagger} \boldsymbol{\xi} \boldsymbol{\xi}^{H} \boldsymbol{H}^{\dagger H} \boldsymbol{G}^{H} \boldsymbol{F}^{H}\right\}
$$

and investigate the influence of $M$ and $L$ on the estimation accuracy. For the proof of (20) we refer to [12], and its references, where the related problem of estimating sinusoids in noise, is studied.

Now, we show that the order of magnitude of $\mathcal{P}_{t}$ is $O\left(1 / R_{t}^{2} R_{f}^{3}\right)$ for sufficiently large $M$ and when $L$ is of the order of $M$. First, notice that $H$ can be factorized as follows:

$$
\begin{aligned}
\boldsymbol{H}= & \sum_{k=0}^{K-1} c_{k}\left(\begin{array}{ccc}
u_{k}^{-1} & \ldots & u_{k}^{-L} \\
\vdots & & \vdots \\
u_{k}^{M} & \ldots & u_{k}^{M-L}
\end{array}\right) \\
= & \left(\begin{array}{cccc}
1 & \ldots & 1 \\
\vdots & & \vdots \\
u_{0}^{M} & \ldots & u_{K-1}^{M}
\end{array}\right)\left(\begin{array}{ccc}
c_{0} & & 0 \\
& \ddots & \\
0 & & c_{K-1}
\end{array}\right) \\
& \times\left(\begin{array}{ccc}
u_{0}^{-1} & \ldots & u_{0}^{-L} \\
\vdots & & \vdots \\
u_{K-1}^{-1} & \ldots & u_{K-1}^{-L}
\end{array}\right)=\boldsymbol{S C G} .
\end{aligned}
$$


Since all these matrices are of full rank, we can use standard results on the Moore-Penrose pseudoinverse together with (21), to show that

$$
\boldsymbol{H}^{\dagger}=\boldsymbol{G}^{H}\left(\boldsymbol{G} \boldsymbol{G}^{H}\right)^{-1} \boldsymbol{C}^{-1}\left(\boldsymbol{S}^{H} \boldsymbol{S}\right)^{-1} \boldsymbol{S}^{H}
$$

$$
\begin{aligned}
& \frac{1}{L^{2}}\left[\cos \left(2 \pi t_{0}\right) \cdots L \cos \left(2 L \pi t_{0}\right)\right] G^{H} \\
& =\left[\begin{array}{llll}
O(1)-j O(1 / L) & O(1 / L)(1-j) & \cdots & O(1 / L)(1-j)
\end{array}\right] .
\end{aligned}
$$

Inserting (22) in (20), we obtain

$$
\mathcal{P}_{t}=\frac{1}{4 \pi^{2}} R e\left\{\boldsymbol{F} \boldsymbol{C}^{-1}\left(\boldsymbol{S}^{H} \boldsymbol{S}\right)^{-1} \boldsymbol{S}^{H} \boldsymbol{\xi} \boldsymbol{\xi}^{H} \boldsymbol{S}\left(\boldsymbol{S}^{H} \boldsymbol{S}\right)^{-1} \boldsymbol{C}^{-1} \boldsymbol{F}^{H}\right\}
$$

In the next steps of the proof, we analyze in detail the different terms involving $\boldsymbol{P}_{t}$ in (23). We examine the order of each term and use the notation $g=\operatorname{diag}\left[O\left(f_{1}(q)\right)\right]+O\left(f_{2}(q)\right)$ to denote that the matrix $g$ has diagonal elements of the order $O\left(f_{1}(q)\right)$ and off-diagonal elements of the order $O\left(f_{2}(q)\right)$ while $f_{1}(q)$ and $f_{2}(q)$ are any two functions of $q$. For evaluating the order of some terms we also need the following standard result [23]

$$
\begin{aligned}
& \frac{1}{M^{s+1}} \sum_{m=1}^{M} m^{s} e^{-j m(\omega-\bar{\omega})} \\
& \quad=\left\{\begin{array}{ll}
O(1) & \text { for } \omega=\bar{\omega} \text { and } s \geq 0 \\
O(1 / M)(1-j) & \text { for } \omega \neq \bar{\omega} \text { and } s \geq 0
\end{array} .\right.
\end{aligned}
$$

1) $\boldsymbol{C}=\operatorname{diag}[O(1)]$ and consequently $\boldsymbol{C}^{-1}=\operatorname{diag}[O(1)]$, since $\boldsymbol{C}$ depends only on the signal $x(t)$ and has no dependence on $M$ or $L$.

2) $(1 / L) \boldsymbol{G G}^{H}=\operatorname{diag}[O(1)]+O(1 / L)(1-j)$ and consequently $\left((1 / L) \boldsymbol{G G}^{H}\right)^{-1}=\operatorname{diag}[O(1)]+O(1 / L)(1-j)$, which can be verified by direct multiplication using (24).

3) $(1 / M) \boldsymbol{S}^{H} \boldsymbol{S}=\operatorname{diag}[O(1)]+O(1)-j O(1 / M)$ and consequently $\left((1 / M) \boldsymbol{S}^{H} \boldsymbol{S}\right)^{-1}=\operatorname{diag}[O(1)]+O(1)-$ $j O(1 / M)$, which can be verified again by direct multiplication since the matrix $\boldsymbol{S}$ has a similar structure as $\boldsymbol{G}^{H}$.

4) $L \boldsymbol{F}=\operatorname{diag}[O(1 / L)+j O(1)]$ and consequently $(L \boldsymbol{F})^{-1}=\operatorname{diag}[O(1 / L)+j O(1)]$ From the definition of $\alpha_{k}$, we have that:

$$
\begin{aligned}
\frac{1}{L} \alpha_{0}= & \frac{1}{L}\left[\begin{array}{lll}
\cos 2 \pi t_{0} & \cdots & L \cos L 2 \pi t_{0}
\end{array}\right] \boldsymbol{A} \\
= & \frac{1}{L}\left[\begin{array}{lll}
\cos 2 \pi t_{0} & \cdots & L \cos L 2 \pi t_{0}
\end{array}\right]\left(-\boldsymbol{H}^{\dagger} \boldsymbol{h}\right) \\
= & \frac{1}{L^{2}}\left[\begin{array}{lll}
\cos 2 \pi t_{0} & \cdots & L \cos L 2 \pi t_{0}
\end{array}\right] \boldsymbol{G}^{H}\left(\frac{1}{L} \boldsymbol{G} \boldsymbol{G}^{H}\right)^{-1} \\
& \times \boldsymbol{C}^{-1}\left(\frac{1}{M} \boldsymbol{S}^{H} \boldsymbol{S}\right)^{-1}\left(\frac{1}{M} \boldsymbol{S}^{H} \boldsymbol{h}\right) .
\end{aligned}
$$

Next, by multiplying the corresponding matrices, it can also be verified, by direct computation, that:

For the last part in (25) we get that

$$
\begin{aligned}
\frac{1}{M} \boldsymbol{S}^{H} \boldsymbol{h} & =\left(\begin{array}{ccc}
u_{0}^{0} & \ldots & u_{K-1}^{0} \\
\vdots & & \vdots \\
u_{0}^{M} & \ldots & u_{K-1}^{M}
\end{array}\right)^{H}\left(\begin{array}{c}
\frac{1}{\tau} \sum_{k=0}^{K-1} c_{k} u_{k}^{0} \\
\vdots \\
\frac{1}{\tau} \sum_{k=0}^{K-1} c_{k} u_{k}^{M}
\end{array}\right) \\
& =\frac{1}{M}\left(\begin{array}{c}
\sum_{m=0}^{M} \sum_{k=0}^{K-1} c_{k} u_{k}^{m} u_{0}^{-m} \\
\vdots \\
\sum_{m=0}^{M} \sum_{k=0}^{K-1} c_{k} u_{k}^{m} u_{K-1}^{-m}
\end{array}\right) .
\end{aligned}
$$

The term on the right-hand side (RHS) of the previous equation has the following order:

$$
\begin{aligned}
\frac{1}{M} \sum_{m=0}^{M} \sum_{k=0}^{K-1} c_{k} u_{k}^{m} u_{r}^{-m} \\
=\frac{1}{M} \sum_{k=0}^{K-1} c_{k}+\frac{1}{M} \sum_{m=1}^{M} \sum_{k=0}^{K-1} c_{k} e^{-j \frac{2 \pi}{\tau} m\left(t_{k}-t_{r}\right)} \\
=\frac{1}{M} \sum_{k=0}^{K-1} c_{k}+\frac{1}{M} \sum_{m=1}^{M} c_{r} \\
\quad+\frac{1}{M} \sum_{m=1}^{M} \sum_{k=0, k \neq r}^{K-1} c_{k} e^{-j \frac{2 \pi}{\tau} m\left(t_{k}-t_{r}\right)} \\
=O(1 / M)+O(1) \\
\quad+\sum_{k=0, k \neq r}^{K-1} c_{k}(O(1 / M)+j O(1 / M)) \\
=O(1)+j O(1 / M)
\end{aligned}
$$

and, therefore

$$
\frac{1}{M} \boldsymbol{S}^{H} \boldsymbol{h}=[O(1)+j O(1 / M)]_{K \times 1} \cdot
$$

Putting together the results from previous steps and substituting (26) and (27) in (25) and assuming that $L$ and $M$ are of the same order, we can conclude that

$$
\frac{1}{L} \alpha_{0}=O(1)+j O(1 / L)
$$


Similarly:

$\frac{1}{L^{2}}\left[\begin{array}{lll}\sin \left(2 \pi t_{0}\right) & \cdots & L \sin \left(2 L \pi t_{0}\right)\end{array}\right] G^{T}$
$\quad=\left[\begin{array}{llll}O(1 / L)-j O(1) & O(1 / L)(1-j) & \cdots & O(1 / L)(1-j)\end{array}\right]$,

and we obtain

$$
\frac{1}{L} \beta_{0}=O(1 / L)+j O(1)
$$

The same procedure can be used to evaluate the order of $\left\{\alpha_{k}, \beta_{k}\right\}$ for $k \geq 0$, obtaining

$\frac{1}{L} \alpha_{k}=O(1)+j O(1 / L)$ and $\frac{1}{L} \beta_{k}=O(1 / L)+j O(1)$.

This leads to the conclusion that $L \boldsymbol{F}=\operatorname{diag}[O(1 / L)+$ $j O(1)]$.

5) $\boldsymbol{S}^{H} \boldsymbol{\xi} \boldsymbol{\xi}^{H} \boldsymbol{S}=\operatorname{MSE}(\boldsymbol{X}, \hat{\boldsymbol{X}}) O(L)=\operatorname{MSE}\left(\boldsymbol{Y}_{\mathbf{2}}, \hat{\boldsymbol{Y}}_{\mathbf{2}}\right)$ for $M \geq L$

The key point is that first we can split matrix $\boldsymbol{\xi} \xi^{H}$ into two terms, one depending only on $R_{t}$, and the other one depending only on $M$ and $L$, or equivalently, on $R_{f}$, assuming that $L$ is also of the order of $M$. Following similar arguments as in [12], the matrix $\boldsymbol{\xi} \boldsymbol{\xi}^{H}$ can be rewritten as:

$$
\boldsymbol{\xi} \boldsymbol{\xi}^{H}=\operatorname{MSE}\left(\boldsymbol{Y}_{2}, \hat{\boldsymbol{Y}}_{2}\right) A^{*} A^{T},
$$

where $(\cdot)^{*}$ denotes conjugation of $(\cdot)$ and

$$
A=\left(\begin{array}{cccccc}
1 & a_{1} & \cdots & a_{L} & & 0 \\
& \ddots & \ddots & & \ddots & \\
& 0 & 1 & a_{1} & \cdots & a_{L}
\end{array}\right)_{(M+1 \mid M+L+1)}
$$

Thus, it follows that

$$
\boldsymbol{S}^{H} \boldsymbol{\xi} \boldsymbol{\xi}^{H} \boldsymbol{S}=\operatorname{MSE}\left(\boldsymbol{Y}_{\mathbf{2}}, \hat{\boldsymbol{Y}}_{\mathbf{2}}\right) \boldsymbol{S}^{H} A^{*} A^{T} \boldsymbol{S} .
$$

In order to obtain an insight on the order of $\boldsymbol{S}^{H} \boldsymbol{\xi} \boldsymbol{\xi}^{H} \boldsymbol{S}$, we compute the explicit form for the case of one Dirac and describe how to generalize the result for the case of $K$ Diracs.

For $K=1$, we have that

$$
\begin{aligned}
& \boldsymbol{S}=\left(\begin{array}{llll}
u_{0}^{0} & u_{0}^{1} & \cdots & u_{0}^{M}
\end{array}\right)^{T}, \quad \boldsymbol{S}^{H} \boldsymbol{S}=M+1, \quad \boldsymbol{G} \boldsymbol{G}^{H}=L, \\
& \boldsymbol{a}=-\boldsymbol{H}^{\dagger} c_{0}\left(\begin{array}{c}
u_{0}^{0} \\
u_{0}^{1} \\
\vdots \\
u_{0}^{M}
\end{array}\right)=-\boldsymbol{G}^{H}\left(\boldsymbol{G} \boldsymbol{G}^{H}\right)^{-1} \boldsymbol{C}^{-1}\left(\boldsymbol{S}^{H} \boldsymbol{S}\right)^{-1} \boldsymbol{S}^{H} \boldsymbol{S} c_{0} \\
& =-\frac{1}{L}\left(\begin{array}{c}
u_{0}^{1} \\
\vdots \\
u_{0}^{L}
\end{array}\right) \text {. }
\end{aligned}
$$

Then, for the case where $M \geq L$, we have that

$$
\begin{aligned}
A^{T} \boldsymbol{S} & =\left(\begin{array}{ccc}
1 & & \\
-\frac{1}{L} u_{0} & & 0 \\
\vdots & \ddots & \\
-\frac{1}{L} u_{0}^{L} & & 1 \\
& \ddots & -\frac{1}{L} u_{0} \\
0 & & \vdots \\
& & -\frac{1}{L} u_{0}^{L}
\end{array}\right)\left(\begin{array}{c}
u_{0}^{0} \\
u_{0}^{1} \\
\vdots \\
u_{0}^{M}
\end{array}\right) \\
& =\left(\begin{array}{c}
u_{0}\left(1-\frac{1}{L}\right) \\
\vdots \\
u_{0}^{L-1}\left(1-\frac{L-1}{L}\right) \\
0 \\
\vdots \\
0 \\
-u_{0}^{M+1} \frac{L}{L} \\
\vdots \\
-u_{0}^{M+L} \frac{1}{L}
\end{array}\right)
\end{aligned}
$$

and

$$
\begin{aligned}
\boldsymbol{S}^{H} A^{*} A^{T} \boldsymbol{S}= & 1+\left(1-\frac{1}{L}\right)^{2}+\cdots+\left(1-\frac{L-1}{L}\right)^{2}+1 \\
& +\left(\frac{L-1}{L}\right)^{2}+\cdots+\left(\frac{1}{L}\right) \\
= & \frac{2}{L^{2}} \sum_{i=0}^{L} i^{2}=\frac{(L+1)(2 L+1)}{3 L}
\end{aligned}
$$


Now, noticing that the coefficients of the annihilating filter have the following form:

$$
\begin{aligned}
\boldsymbol{a} & =-\boldsymbol{H}^{\dagger} \boldsymbol{S}\left(\begin{array}{c}
c_{0} \\
\vdots \\
c_{K-1}
\end{array}\right) \\
& =-\left(\boldsymbol{G}^{H}\left(\boldsymbol{G} \boldsymbol{G}^{H}\right)^{-1} \boldsymbol{C}^{-1}\left(\boldsymbol{S}^{H} \boldsymbol{S}\right)^{-1} \boldsymbol{S}^{H}\right) \boldsymbol{S}\left(\begin{array}{c}
c_{0} \\
\vdots \\
c_{K-1}
\end{array}\right) \\
& =-\boldsymbol{G}^{H}\left(\boldsymbol{G}^{H}\right)^{-1}\left(\begin{array}{c}
1 \\
\vdots \\
1
\end{array}\right) \\
& =-\frac{1}{L}\left(\begin{array}{c}
\sum_{k=0}^{K-1} u_{k}^{1}+O(1 / L) \\
\sum_{k=0}^{K-1} u_{k}^{2}+O(1 / L) \\
\vdots \\
\sum_{k=0}^{K-1} u_{k}^{L}+O(1 / L)
\end{array}\right)
\end{aligned}
$$

then, by doing the same steps as for the case of one Dirac, we get that the diagonal elements of $\boldsymbol{S}^{H} A^{*} A^{T} \boldsymbol{S}$ are of the order $\mathrm{O}(\mathrm{L})$, or equivalently

$$
\begin{aligned}
\frac{1}{L}(\cdot)_{i i} & =\frac{1}{L}\left(\begin{array}{llll}
u_{i}^{0} & u_{i}^{-1} & \cdots & u_{i}^{-M}
\end{array}\right) A^{*} A^{T}\left(\begin{array}{c}
u_{i}^{0} \\
u_{i}^{1} \\
\vdots \\
u_{i}^{M}
\end{array}\right) \\
& =O(1)+O(1 / L) .
\end{aligned}
$$

For the off-diagonal elements, taking into account the specific structure of $A^{*} A^{T}$ that follows from (30), we have

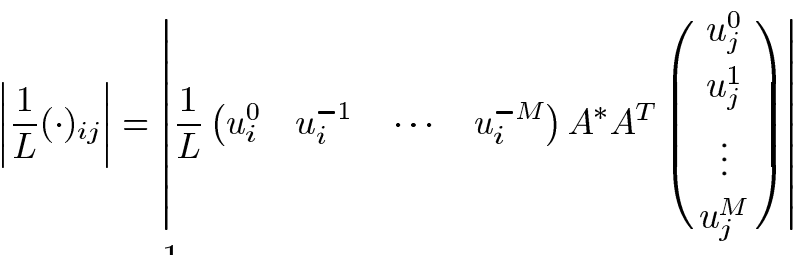

$$
\begin{aligned}
& \leq \frac{1}{L}(\cdot)_{i i}=O(1)+O(1 / L) .
\end{aligned}
$$

This upper bound is sufficient for us and we do not have to search for tighter bounds. Thus

$$
\boldsymbol{S}^{H} A^{*} A^{T} \boldsymbol{S}=O(L)
$$

Finally, we can calculate the order of the matrix $\mathcal{P}_{t}$ as follows:

$$
\begin{aligned}
\mathcal{P}_{t}= & \frac{1}{2} \frac{1}{4 \pi^{2}} \frac{1}{L^{2} M^{2}} \operatorname{Re}\left\{(L \boldsymbol{F}) \boldsymbol{C}^{-1}\left(\frac{1}{M} \boldsymbol{S}^{T} \boldsymbol{S}\right)^{-1}\left(\boldsymbol{S}^{T} \boldsymbol{\xi} \boldsymbol{\xi}^{T} \boldsymbol{S}\right)\right. \\
& \left.\times\left(\frac{1}{M} \boldsymbol{S}^{T} \boldsymbol{S}\right)^{-1} \boldsymbol{C}^{-1}(L \boldsymbol{F})^{H}\right\} \\
= & \frac{1}{2} \frac{1}{4 \pi^{2}} \frac{L}{L^{2} M^{2}} \operatorname{MSE}\left(\boldsymbol{Y}_{\mathbf{2}}, \hat{\boldsymbol{Y}}_{\mathbf{2}}\right)[O(1)+j O(1)]_{K \times K} \\
= & \frac{1}{L M^{2}} d_{1}(\boldsymbol{y}, \hat{\boldsymbol{y}})[O(1)+j O(1)]_{K \times K} .
\end{aligned}
$$

Changing $L=M=O\left(R_{f}\right)$ in (32) and using the result from Theorem 1 for $\boldsymbol{y} \in \boldsymbol{S}_{\mathbf{1}} \cap \boldsymbol{S}_{\mathbf{2}}$ we get that:

$$
\mathcal{P}_{t}=O\left(\frac{1}{R_{t}^{2} R_{f}^{3}}\right)
$$

The final results follows from [24], where it is shown that the results derived for the Yule-Walker system and the least square solution has asymptotically the same behavior as the total least square solution. Therefore

$$
d_{3}(\boldsymbol{y}, \hat{\boldsymbol{y}})=\operatorname{MSE}(\boldsymbol{t}, \hat{\boldsymbol{t}})=\sum_{i=1}^{K}\left(\mathcal{P}_{t}\right)_{i i}=O\left(\frac{1}{R_{t}^{2} R_{f}^{3}}\right)
$$

\section{ACKNOWLEDGMENT}

The authors wish to thank Dr. N. T. Thao for very helpful comments that improved substantially the clarity of the paper. They would like also to thank the anonymous reviewers for their very valuable comments.

\section{REFERENCES}

[1] M. Vetterli, P. Marziliano, and T. Blu, "Sampling signals with finite rate of innovation," IEEE Trans. Signal Process., vol. 50, no. 6, pp. 1417-1428, Jun. 2002.

[2] I. Maravić and M. Vetterli, "Digital DS-CDMA receiver working below the chip rate: Theory and design,", Tech. Rep. IC/2002/018, 2002.

[3] I. Maravić, M. Vetterli, and K. Ramchandran, "High-resolution acquisition methods for wideband communication systems," in Proc. Int. Conf. Acoustics, Speech, Signal Processing (ICASSP), vol. 4, Apr. 2003, pp. $133-136$.

[4] P. Ishwar, A. Kumar, and K. Ramchandran, "Distributed sampling for dense sensor networks: A bit-conservation principle," presented at the Symp. Information Processing Sensor Networks (IPSN), Palo Alto, CA, 2003.

[5] B. Beferull-Lozano, R. L. Konsbruck, and M. Vetterli, "Rate-distortion problem for physics based distributed sensing," presented at the 3rd Int. Symp. Information Processing Sensor Networks (IPSN), Berkeley, CA, Apr. 2004.

[6] N. T. Thao and M. Vetterli, "Deterministic analysis of oversampled $A / D$ conversion and decoding improvement based on consistent estimates," Trans. Signal Process., vol. 42, no. 3, pp. 519-531, Mar. 1994. 
[7] B. Beferull-Lozano and A. Ortega, "Efficient quantization for overcomplete expansion in $R^{N}$," IEEE Trans. Inf. Theory, vol. 49, no. 1, pp. 129-150, Jan. 2003.

[8] V. K. Goyal, M. Vetterli, and N. T. Thao, "Quantized overcomplete expansion in $R^{N}$ : Analysis, sinthesis and algorithms," IEEE Trans. Inf. Theory, vol. 44, no. 1, pp. 16-31, Jan. 1998.

[9] I. Jovanović and B. Beferull-Lozano, "Oversampled $A / D$ conversion of nonbandlimited signals with finite rate of innovation," presented at the IEEE Int. Conf. Acoust., Speech, Signal Processing (ICASSP), Montreal, QC, Canada, May 2004.

[10] Z. Cvetković and M. Vetterli, "On simple oversampled $A / D$ conversion in $L^{2}(\mathcal{R})$," IEEE Trans. Inf. Theory, vol. 47, no. 1, pp. 146-154, 2001.

[11] I. Jovanović and B. Beferull-Lozano, "Error-rate dependence of nonbandlimited signals with finite rate of innovation," presented at the IEEE Int. Symp. Inf. Theory (ISIT), Chicago, IL, Jun. 2004.

[12] P. Stoica, T. Soderstorm, and F. Ti, "Asymptotic properties of the highorder Yule Walker estimqtes of sinusoidal frequencies," IEEE Trans. Acoust., Speech, Signal Process., vol. 37, no. 11, pp. 1721-1734, Nov. 1989.

[13] S. Van Huffel and J. Vandewalle, The Total Least Squares Problem: Computational Aspects and Analysis. Philadelphia, PA: SIAM, 1991, vol. 9, Frontiers in Applied Mathematics Series.

[14] P. Stoica, B. Friedlander, and T. Soderstorm, "A high-order yule-walker method of ARparameters of ARMA model," Syst. Contr. Lett., vol. 11, pp. 99-105, 1989.

[15] T. K. Moon and W. C. Stirling, Mathematical Methods and Algorithms for Signal Processing. Englewood Cliffs, NJ: Prentice-Hall, 2000.

[16] Z. Cvetković and I. Daubechies, "Single-bit oversampled $A / D$ conversion with exponential accuracy in the bit-rate," presented at the Data Compress. Conf. (DCC), Snowbird, UT, 2000.

[17] Z. Cvetković, "Resilience properties of redundant expansions under additive noise and quantization," IEEE Trans. Inf. Theory, vol. 49, no. 3, pp. 644-656, Mar. 2003.

[18] — "Source coding with quantized redundant expansions: Accuracy and reconstruction," presented at the Data Compression Conf. (DCC), Snowbird, UT, 1999

[19] N. T. Thao and M. Vetterli, "Reduction of the MSE in r-times oversampled $A / D$ conversion from $O(1 / r)$ to $O\left(1 / r^{2}\right)$," IEEE Trans. Signal Process., vol. 42, no. 1, pp. 200-203, Jan. 1994.

[20] R. J. Duffin and A. C. Schaeffer, "A class of nonharmonic Fourier series," Trans. Amer. Math. Soc., vol. 72, pp. 341-366, 1952.

[21] H. J. Landau, "Sampling, data transmission, and the Nyquist rate," Proc. IEEE, vol. 55, pp. 200-2003, 1994.

[22] T. Soderstorm and P. Stoica, "Accuracy of high-order Yule-Walker methods for frequency estimation of complex sine waves," Inst. Elect. Eng. Proc.-F, vol. 140, no. 1, pp. 71-80, 1993.

[23] I. S. Gradshteyn and I. M. Ryzhik, Table of Integrals, Series and Products. New York: Academic, 1980.

[24] P. Stoica, T. Soderstorm, and S. Van Huffel, "On SVD-based and TLSbased high-order Yule-Walker methods for frequency estimation," Signal Process., vol. 29, pp. 309-317, 1992.

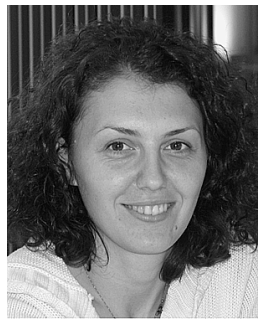

Ivana Jovanović (S'06) received the B.Sc. and M.Sc. degrees from the Faculty of Electrical Engineering, University of Belgrade, Belgrade, Serbia and Montenegro, in 2002

She is currently pursuing the Ph.D. degree in communication systems at the Swiss Federal Institute of Technology (EPFL), Lausanne. From 2000 through 2002, she was with the Electronic Laboratory, Faculty of Electrical Engineering, University of Belgrade, as an assistant for undergraduate students. Since 2003, she has been a Teaching Assistant with the Laboratory for Audio-Visual Communication (EPFL) for classes in digital signal processing and advance signal processing. Her research interests include signal processing for communications, sampling theory, and acoustic tomography.

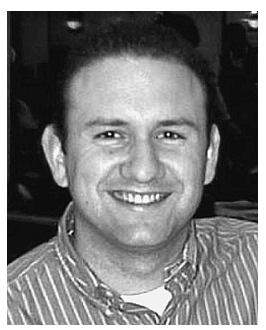

Baltasar Beferull-Lozano (S'01-M'02) was born in Valencia, Spain, in 1972. He received the M.Sc. degree in physics from the Universidad de Valencia, in 1995 (First in Class Honors) and the M.Sc. and Ph.D. degrees in electrical engineering from the University of Southern California (USC), Los Angeles, in 1999 and 2002, respectively. His Ph.D. work was supported by a National Graduate Doctoral Fellowship from the Ministry of Education of Spain.

From January 1996 to August 1997, he was a Research Fellow Assistant with the Department of Electronics and Computer Science, Universidad de Valencia, and from September 1997 to September 2002, he was a Research Fellow Assistant with the Department of Electrical Engineering, the NSF Research Integrated Media Systems Center, and the Signal and Image Processing Institute (SIPI), at USC. He was also with AT\&T Shannon Laboratories (formerly AT\&T Bell Laboratories), Information Sciences Center, Florham Park, NJ. From October 2002 to June 2005, he was a Research Associate with the Department of Communication Systems, Swiss Federal Institute of Technology (EPFL), Lausanne, Switzerland, and a Senior Researcher with the Swiss National Competence Center in Research on Mobile Information and Communication Systems. From July to November 2005, he was a Visiting Professor at Universidad de Valencia and Universidad Politécnica de Valencia. Since December 2005, he has been a Research Professor in Instituto de Robótica-Escuela Técnica Superior de Ingeniería at Universidad de Valencia, and he is the Head of the Group of Information and Communication Systems. His research interests are in the general areas of signal and image processing, distributed signal processing and communications for wireless networks, information theory, and communication theory.

Dr. Beferull-Lozano has served as a member of the technical program committees for several ACM and IEEE international conferences. At USC, he received several awards, including the Best Ph.D. Thesis paper Award in April 2002 and the Outstanding Academic Achievement Award in April 1999 and April 2002. He has served as a member of the Technical Program Committees for several ACM and IEEE International Conferences. 